\title{
A ROBUST ITERATIVE APPROACH FOR SOLVING NONLINEAR VOLTERRA DELAY INTEGRO-DIFFERENTIAL EQUATIONS
}

\author{
Austine Efut Ofem \\ Department of Mathematics, University of Uyo, \\ Uyo, Nigeria \\ ofemaustine@gmail.com \\ Unwana Effiong Udofia \\ Department of Mathematics and Statistics, Akwa Ibom State University, \\ Ikot Akpaden, Mkpatenin, Nigeria \\ unwanaudofia.aksu@yahoo.com

\section{Donatus Ikechi Igbokwe} \\ Department of Mathematics, Michael Okpara University of Agriculture, \\ Umudike, Nigeria \\ igbokwedi@yahoo.com
}

\begin{abstract}
This paper presents a new iterative algorithm for approximating the fixed points of multivalued generalized $\alpha$-nonexpansive mappings. We study the stability result of our new iterative algorithm for a larger concept of stability known as weak $w^{2}$-stability. Weak and strong convergence results of the proposed iterative algorithm are also established. Furthermore, we show numerically that our new iterative algorithm outperforms several known iterative algorithms for multivalued generalized $\alpha$-nonexpansive mappings. Again, as an application, we use our proposed iterative algorithm to find the solution of nonlinear Volterra delay integro-differential equations. Finally, we provide an illustrative example to validate the mild conditions used in the result of the application part of this study. Our results improve, generalize and unify several results in the existing literature.
\end{abstract}

Keywords: Banach space, Uniformly convex Banach space, Multivalued generalized $\alpha$-nonexpansive mapping, Convergence, Nonlinear Volterra delay integro-differential equations.

\section{Introduction}

A mapping $g$ on a nonempty subset $\mathcal{K}$ of a Banach $\mathscr{G}$ is called nonexpansive if

$$
\|g(x)-g(y)\| \leq\|x-y\|, \quad \text { for all } \quad x, y \in \mathcal{K} .
$$

A point $x$ in $\mathcal{K}$ is said to be a fixed point of $g$ if $g(x)=x$. We denote the set of all fixed points of $g$ by

$$
\mathcal{F}(g)=\{x \in \mathcal{K}: x=g(x)\} .
$$

Let $\mathbb{R}$ denote the set of all real numbers and $\mathbb{N}$ be the set of all natural numbers.

In 1965, Browder [7], Göhde [10] and Kirk [17] independently studied the existence of fixed points of nonexpansive mappings in Banach spaces. The authors showed that every nonexpansive 
mapping defined on a bounded closed convex subset of a uniformly convex Banach space always has a fixed point. Recently, many authors have introduced and studied some new classes of mappings which are considered to be larger than the single-valued nonexpansive mappings.

One of the first extensions and generalizations of single-valued nonexpansive mapping which has fascinated many authors was introduced by Suzuki [33] in 2008. Such mappings are generally known as mappings satisfying condition (C). The author proved some existence and convergence results for such mapping.

Definition 1. A mapping $g: \mathcal{K} \rightarrow \mathcal{K}$ is said to be Suzuki generalized nonexpansive mapping or mapping satisfying condition $(C)$ if for all $x, y \in \mathcal{K}$ we have

$$
\frac{1}{2}\|x-g x\| \leq\|x-y\| \quad \text { implies } \quad\|g x-g y\| \leq\|x-y\| .
$$

In 2011, Aoyama and Kohshaka [5] introduced a new class of single-valued mappings known as $\alpha$-nonexpansive mappings and obtained some fixed point theorems for such mappings.

Definition 2. A mapping $g: \mathcal{K} \rightarrow \mathcal{K}$ is said to be an $\alpha$-nonexpansive with $\alpha \in[0,1)$ if

$$
\|g x-g y\|^{2} \leq \alpha\|g x-y\|^{2}+\alpha\|g y-x\|^{2}+(1-2 \alpha)\|x-y\|^{2},
$$

for all $x, y \in \mathcal{K}$.

Obviously, every nonexpansive mapping is an $\alpha$-nonexpansive with $\alpha=0$ (i.e., 0 -nonexpansive mapping).

In 2017, Pant and Shukla [29] introduced a new type of single-valued nonexpansive mappings known as generalized $\alpha$-nonexpansive mappings and obtained some existence and convergence theorems.

Definition 3. A mapping $g: \mathcal{K} \rightarrow \mathcal{K}$ is said to be generalized $\alpha$-nonexpansive with $\alpha \in[0,1)$ if

$$
\begin{gathered}
\frac{1}{2}\|x-g x\| \leq\|x-y\| \quad \text { implies } \\
\|g x-g y\| \leq \alpha\|g x-y\|+\alpha\|g y-x\|+(1-2 \alpha)\|x-y\|,
\end{gathered}
$$

for all $x, y \in \mathcal{K}$.

This class of mappings properly includes nonexpansive and Suzuki generalized nonexpansive mappings [29].

Fixed point theory for multivalued mappings has useful applications in control theory, convex optimization, differential equations and economics. The fixed points of multivalued mappings were first studied by Markin [20] and Nadler [21].

A set $\mathcal{K}$ is said to be proximinal if for each $x \in \mathscr{G}$, there exists an element $y \in \mathcal{K}$ such that $\|x-y\|=d(x, \mathcal{K})$, where

$$
d(x, \mathcal{K})=\inf \{\|x-\ell\|: \ell \in \mathcal{K}\} .
$$

We denote by $C B(\mathcal{K}), C(\mathcal{K})$ and $\mathcal{P}(\mathcal{K})$ the families of nonempty closed and bounded subsets, nonempty compact subsets and nonempty proximinal subsets of $\mathcal{K}$, respectively. Let $\mathscr{H}$ be the Hausdorff metric induced by $d$ of $\mathscr{G}$ which is defined as:

$$
\mathscr{H}(\mathscr{U}, \mathscr{V})=\max \left\{\sup _{x \in \mathscr{U}}(x, \mathscr{V}), \sup _{y \in \mathscr{V}}(y, \mathscr{U})\right\}, \quad \text { for all } \quad \mathscr{U}, \mathscr{V} \in \mathrm{CB}(\mathcal{K}) .
$$

An element $x \in \mathcal{K}$ is said to be a fixed point of a multivalued mapping $\mathcal{T}: \mathcal{K} \rightarrow \mathcal{P}(\mathcal{K})$ if $x \in \mathcal{T} x$. Let $F(\mathcal{T})=\{x \in \mathcal{K}: x \in \mathcal{T} x\}$ denote the set of all fixed points of $\mathcal{T}$. 
A multivalued mapping $\mathcal{T}: \mathcal{K} \rightarrow \mathcal{P}(\mathcal{K})$ is said to be a contraction if there exists a constant $\delta \in[0,1)$ such that for all $x, y \in \mathcal{K}$,

$$
\mathscr{H}(\mathcal{T} x, \mathcal{T} y) \leq \delta\|x-y\|,
$$

and nonexpansive if

$$
\mathscr{H}(\mathcal{T} x, \mathcal{T} y) \leq\|x-y\|
$$

for all $x, y \in \mathcal{K}$. The study of fixed points for multivalued contraction and nonexpansive mappings using the Hausdorff metric was initiated by Markin [20].

In 2011, Abkar and Eslamian [2] gave the multivalued version of Suzuki generalized nonexpansive mappings.

Definition 4. A multivalued mapping $\mathcal{T}: \mathcal{K} \rightarrow C B(\mathcal{K})$ is said to be Suzuki generalized nonexpansive mappings or said to satisfy condition $(C)$ if for all $x, y \in \mathcal{K}$, we have

$$
\frac{1}{2} d(x, \mathcal{T} x) \leq\|x-y\| \quad \text { implies } \quad \mathscr{H}(\mathcal{T} x, \mathcal{T} y) \leq\|x-y\| .
$$

Recently, Iqbal et al. [15] introduced a multivalued generalized $\alpha$-nonexpansive mapping and obtained some fixed points results in uniformly convex Banach spaces.

Definition 5. A mapping $\mathcal{T}: \mathcal{K} \rightarrow C B(\mathcal{K})$ is said to be a multivalued generalized $\alpha$ nonexpansive if there exists $\alpha \in[0,1)$ such that

$$
\begin{gathered}
\frac{1}{2} d(x, \mathcal{T} x) \leq\|x-y\| \quad \text { implies } \\
\mathscr{H}(\mathcal{T} x, \mathcal{T} y) \leq \alpha d(x, \mathcal{T} y)+\alpha d(y, \mathcal{T} x)+(1-2 \alpha)\|x-y\|,
\end{gathered}
$$

for all $x, y \in \mathcal{K}$.

It is not hard to see that every multivalued mapping satisfying condition $(C)$ is multivalued generalized $\alpha$-nonexpansive mapping with $\alpha=0$ and also, every multivalued generalized $\alpha$-nonexpansive mapping with a nonempty fixed point set is multivalued quasi-nonexpansive.

The fixed point theory of the classes of multivalued nonexpansive mappings is more cumbersome than the corresponding theory for the classes of single valued nonexpansive mappings. But the numerous applications of the former have caused several researchers to study not only the existence and uniqueness of fixed points of different classes of multivalued nonexpansive mappings, but also approximated the fixed points of different classes of multivalued nonexpansive mappings.

In the course of approximating the fixed points of the classes of nonexpansive mappings, several iterative algorithms have be introduced and studied. Some of the well known iterative algorithms in existing literature are given in Mann [19], Ishikawa [16], Noor [22], S [3], Abbas and Nazir [1], Tharkur [34] and many more.

In 2009, Shahzad and Zegeye [11] studied convergence of the Mann and Ishikawa iterative algorithms for multivalued nonexpansive mappings in a nonempty closed convex subset of a uniformly convex Banach space. The authors defined

$$
\mathcal{P}_{\mathcal{T}}(x)=\{y \in \mathcal{T} x:\|x-y\|=d(x, \mathcal{T} x)\}
$$

for a multivalued mapping $\mathcal{T}: \mathcal{K} \rightarrow \mathcal{P}(\mathcal{K})$ to make it well defined.

The famous Mann iterative algorithms is defined as:

$$
\left\{\begin{array}{l}
x_{0}=x \in \mathcal{K}, \\
x_{n+1}=\left(1-u_{n}\right) x_{n}+u_{n} \ell_{n}, \quad \forall n \geq 1,
\end{array}\right.
$$


where $\left\{u_{n}\right\}$ is a sequence in $(0,1)$ and $\ell_{n} \in \mathcal{P}_{\mathcal{T}}\left(x_{n}\right)$.

The Ishikawa iterative algorithms is defined as:

$$
\left\{\begin{array}{l}
x_{0}=x \in \mathcal{K}, \\
y_{n}=\left(1-v_{n}\right) x_{n}+v_{n} \ell_{n}, \quad \forall n \geq 1, \\
x_{n+1}=\left(1-u_{n}\right) x_{n}+u_{n} \zeta_{n},
\end{array} \quad \forall\right.
$$

where $\left\{u_{n}\right\}$ and $\left\{v_{n}\right\}$ are sequences in $(0,1), \zeta_{n} \in \mathcal{P}_{\mathcal{T}}\left(y_{n}\right)$ and $\ell_{n} \in \mathcal{P}_{\mathcal{T}}\left(x_{n}\right)$.

The following iterative algorithm which was introduced by Argawal et al. [3], is known as S-iterative algorithm:

$$
\left\{\begin{array}{l}
x_{0}=x \in \mathcal{K}, \\
y_{n}=\left(1-v_{n}\right) x_{n}+v_{n} \ell_{n}, \quad \forall n \geq 1, \\
x_{n+1}=\left(1-u_{n}\right) \ell_{n}+u_{n} \zeta_{n},
\end{array} \quad \forall n\right.
$$

where $\left\{u_{n}\right\}$ and $\left\{v_{n}\right\}$ are sequences in $(0,1), \zeta_{n} \in \mathcal{P}_{\mathcal{T}}\left(y_{n}\right)$ and $\ell_{n} \in \mathcal{P}_{\mathcal{T}}\left(x_{n}\right)$. The authors proved that (1.4) converges at the same rate of the Picard iteration and faster than the Ishikawa iteration for contractions mappings.

In 2018, Gunduz et al. [11] introduced the multivalued version of Thakur iteration process as follows:

$$
\left\{\begin{array}{l}
x_{0}=x \in \mathcal{K}, \\
z_{n}=\left(1-t_{n}\right) x_{n}+t_{n} \ell_{n}, \\
y_{n}=\left(1-v_{n}\right) x_{n}+v_{n} \omega_{n}, \\
x_{n+1}=\left(1-u_{n}\right) \omega_{n}+u_{n} \zeta_{n},
\end{array} \quad \forall n \geq 1\right.
$$

where $\left\{u_{n}\right\},\left\{t_{n}\right\}$ and $\left\{v_{n}\right\}$ are sequences in $(0,1), \zeta_{n} \in \mathcal{P}_{\mathcal{T}}\left(y_{n}\right), \omega_{n} \in \mathcal{P}_{\mathcal{T}}\left(z_{n}\right)$ and $\ell_{n} \in \mathcal{P}_{\mathcal{T}}\left(x_{n}\right)$. The authors proved numerically that (1.5) convergence is faster than each of Mann, Ishikawa, Noor, S, Abass iteration processes.

Recently, Okeke et al. [28] introduced the multivalued version of Picard-Ishikawa hybrid iterative algorithm which was considered in [26] as follows:

$$
\left\{\begin{array}{l}
x_{0}=x \in \mathcal{K}, \\
z_{n}=\left(1-v_{n}\right) x_{n}+v_{n} \ell_{n}, \quad \forall n \geq 1, \\
y_{n}=\left(1-u_{n}\right) x_{n}+u_{n} \omega_{n}, \quad \forall n, \\
x_{n+1}=\zeta_{n},
\end{array}\right.
$$

where $\left\{u_{n}\right\}$ and $\left\{v_{n}\right\}$ are sequences in $(0,1), \zeta_{n} \in \mathcal{P}_{\mathcal{T}}\left(y_{n}\right), \omega_{n} \in \mathcal{P}_{\mathcal{T}}\left(z_{n}\right)$ and $\ell_{n} \in \mathcal{P}_{\mathcal{T}}\left(x_{n}\right)$. The authors proved analytically and numerally that (1.6) converges faster than a number of existing iterative algorithms for quasi-nonexpansive mapping.

On the other hand, a fixed point iteration procedure is said to be stable numerically if small errors or modifications in the data or procedure has small control on the computed value of the fixed point.

The concept of stability of fixed point iteration process was rigorously studied by Harder in her Ph.D thesis which was published in $[13,14]$.

Definition 6 [13, 14]. Let $\mathcal{T}: \mathcal{K} \rightarrow \mathcal{P}(\mathcal{K})$. Define a fixed point iteration algorithm by $x_{n+1}=f\left(\mathcal{T}, x_{n}\right)$ such that $\left\{x_{n}\right\}$ converges to a fixed point $q \in \mathcal{T}$. Let $\left\{t_{n}\right\}$ be an arbitrary sequence in $\mathscr{G}$. Define

$$
\epsilon_{n}=\left\|t_{n}-f\left(\mathcal{T}, t_{n}\right)\right\|, \quad \forall n \geq 1 .
$$

A fixed point iterative algorithm is said to be $\mathcal{T}$-stable if the following condition is fulfilled:

$$
\lim _{n \rightarrow \infty} \epsilon_{n}=0 \quad \text { if and only if } \quad \lim _{n \rightarrow \infty} t_{n}=q .
$$


The notion of stability in Definition 6 has been studied by several authors for both single and multivalued mappings (see [15, 24, 28] and the references in them).

In [6], Berinde showed that the concept of stability in Definition 6 is not precise because of the sequence $\left\{t_{n}\right\}$ which is arbitrary taken. To overcome this limitation, Berinde [6] observed that it would be more natural that $\left\{t_{n}\right\}$ be an approximate sequence of $\left\{x_{n}\right\}$. Therefore, any iteration algorithm which is stable will also be weakly stable but the converse is generally not true.

Definition 7 [6]. Let $\left\{x_{n}\right\} \subset \mathscr{G}$ be a given sequence. Then a sequence $\left\{t_{n}\right\} \subset \mathscr{G}$ is an approximate sequence of $\left\{x_{n}\right\}$ if, for any $k \in \mathbb{N}$, there exists $\eta=\eta(k)$ such that

$$
\left\|x_{n}-t_{n}\right\| \leq \eta, \quad \forall n \geq k
$$

Definition 8 [6]. Let $\mathcal{T}: \mathcal{K} \rightarrow \mathcal{P}(\mathcal{K})$. Let $\left\{x_{n}\right\}$ be a sequence defined by an iterative algorithm with $x_{0} \in \mathscr{G}$ and

$$
x_{n+1}=f\left(\mathcal{T}, x_{n}\right), \quad n \geq 0 .
$$

Let $\left\{x_{n}\right\}$ converge to a fixed point $q$ of $\mathcal{T}$. Suppose for any approximate sequence $\left\{t_{n}\right\} \subset \mathscr{G}$ of $\left\{x_{n}\right\}$

$$
\lim _{n \rightarrow \infty} \epsilon_{n}=\lim _{n \rightarrow \infty}\left\|t_{n+1}-f\left(\mathcal{T}, t_{n}\right)\right\|=0
$$

implies

$$
\lim _{n \rightarrow \infty} t_{n}=q,
$$

then we say that (1.7) is weakly $\mathcal{T}$-stable or weakly stable with respect to $\mathcal{T}$.

In 2010, Timis [35] studied a new concept of weak stability which is obtained from Definition 8 by replacing of the approximate sequence with the notion of the equivalent sequence which is more general.

Definition 9 [8]. Let $\left\{x_{n}\right\}$ and $\left\{t_{n}\right\}$ be two sequences. We say that these sequences are equivalent if

$$
\lim _{n \rightarrow \infty}\left\|x_{n}-t_{n}\right\|=0
$$

Definition 10 [35]. Let $\mathcal{T}: \mathcal{K} \rightarrow \mathcal{P}(\mathcal{K})$. Let $\left\{x_{n}\right\}$ be an iterative algorithm defined for $x_{0} \in \mathscr{G}$ and

$$
x_{n+1}=f\left(\mathcal{T}, x_{n}\right), \quad n \geq 0 .
$$

Let $\left\{x_{n}\right\}$ converge to a fixed point $q$ of $\mathcal{T}$. Suppose for any equivalent sequence $\left\{t_{n}\right\} \subset \mathscr{G}$ of $\left\{x_{n}\right\}$

$$
\lim _{n \rightarrow \infty} \epsilon_{n}=\lim _{n \rightarrow \infty}\left\|t_{n+1}-f\left(\mathcal{T}, t_{n}\right)\right\|=0
$$

implies

$$
\lim _{n \rightarrow \infty} t_{n}=q
$$

then we shall say that (1.8) is weak $w^{2}$-stable with respect to $\mathcal{T}$. 
Interestingly, the concept of $w^{2}$-stability has not been consumed by many authors for multivalued mappings.

Motivated by the above results, firstly, we construct a new four step iterative algorithm for approximating the fixed points of multivalued generalized $\alpha$-nonexpansive mappings as follows:

$$
\left\{\begin{array}{l}
x_{0}=x \in \mathcal{K}, \\
s_{n}=\left(1-v_{n}\right) x_{n}+v_{n} \ell_{n}, \\
z_{n}=\left(1-u_{n}\right) \ell_{n}+u_{n} h_{n}, \quad \forall n \geq 1, \\
y_{n} \in \mathcal{P}_{\mathcal{T}}\left(z_{n}\right), \\
x_{n+1}=\zeta_{n},
\end{array}\right.
$$

where $\left\{u_{n}\right\}$ and $\left\{v_{n}\right\}$ are sequences in $(0,1), \zeta_{n} \in \mathcal{P}_{\mathcal{T}}\left(y_{n}\right), h_{n} \in \mathcal{P}_{\mathcal{T}}\left(s_{n}\right)$ and $\ell_{n} \in \mathcal{P}_{\mathcal{T}}\left(x_{n}\right)$.

Secondly, we will show that our new iterative algorithm (1.9) is $w^{2}$-stable with respect to $\mathcal{T}$. The stability results are supported with some illustrative examples.

Thirdly, we prove the weak and strong convergence results of the iterative algorithms (1.9) for multivalued generalized $\alpha$-nonexpansive mappings in Banach spaces. Furthermore, a numerical experiment is performed to show that the iterative algorithm (1.9) enjoys a better speed of convergence than all of the iterative processes (1.2)-(1.6) for multivalued generalized $\alpha$-nonexpansive mappings.

Finally, as an application, we will utilize the new iterative method (1.9) to find the solutions of nonlinear Volterra delay integro-differential equations in Banach spaces. An example is also provided to show that our results are applicable.

\section{Preliminaries}

The following definitions, propositions and lemmas will be useful in proving our main results.

Definition 11. A Banach space $\mathscr{G}$ is said to be uniformly convex if for each $\epsilon \in(0,2]$, there exists $\delta>0$ such that for $x, y \in \mathscr{G}$ satisfying $\|x\| \leq 1,\|y\| \leq 1$ and $\|x-y\|>\epsilon$, we have

$$
\left\|\frac{x+y}{2}\right\|<1-\delta
$$

Definition 12. A Banach space $\mathscr{G}$ is said to satisfy Opial's condition if for any sequence $\left\{x_{n}\right\}$ in $\mathscr{G}$ which converges weakly to $x \in \mathscr{G}$ implies

$$
\limsup _{n \rightarrow \infty}\left\|x_{n}-x\right\|<\limsup _{n \rightarrow \infty}\left\|x_{n}-y\right\|, \quad \forall y \in \mathscr{G} \quad \text { with } \quad y \neq x .
$$

Definition 13. Let $\mathscr{G}$ be a Banach space and $\mathcal{K}$ a nonempty closed convex subset of $\mathscr{G}$. Let $\left\{x_{n}\right\}$ be a bounded sequence in $\mathscr{G}$. For $x \in \mathscr{G}$, we put

$$
r\left(x,\left\{x_{n}\right\}\right)=\limsup _{n \rightarrow \infty}\left\|x_{n}-x\right\| .
$$

The asymptotic radius of $r\left(\left\{x_{n}\right\}\right)$ relative to $\left\{x_{n}\right\}$ is defined by

$$
r\left(\mathcal{K},\left\{x_{n}\right\}\right)=\inf \left\{r\left(x,\left\{x_{n}\right\}\right): x \in \mathcal{K}\right\} .
$$

The asymptotic center of $A\left(\left\{x_{n}\right\}\right)$ relative to $\left\{x_{n}\right\}$ is given as:

$$
A\left(\mathcal{K},\left\{x_{n}\right\}\right)=\left\{x \in \mathcal{K}: r\left(x,\left\{x_{n}\right\}\right)=r\left(\mathcal{K},\left\{x_{n}\right\}\right)\right\} .
$$


In a uniformly convex Banach space, it is well known that $A\left(\mathcal{K},\left\{x_{n}\right\}\right)$ consists of exactly one point.

Definition 14. A multivalued mapping $T: \mathcal{K} \rightarrow \mathcal{P}(\mathcal{K})$ is said to be demiclosed at $y \in \mathcal{K}$ if for any sequence $\left\{x_{n}\right\} \in \mathcal{K}$ weakly convergent to $x$ and $y_{n} \in \mathcal{T} x_{n}$ strongly convergent to $y$, we have $y \in \mathcal{T} x$.

Definition 15 [31]. A multivalued mapping $\mathcal{T}: \mathcal{K} \rightarrow C B(\mathcal{K})$ is said to satisfy condition $(I)$ if a nondecreasing function $f:[0, \infty) \rightarrow[0, \infty)$ exists with $f(0)=0$ and for all $r>0$ then $f(r)>0$ such that $d(x, \mathcal{T} x) \geq f(d(x, F(\mathcal{T}))))$, for all $x \in \mathcal{K}$, where

$$
d(x, F(\mathcal{T}))=\inf _{z \in F(\mathcal{T})}\|x-z\| .
$$

Lemma 1 [15]. Let $\mathcal{K}$ be a nonempty subset of a Banach space $\mathscr{G}$ and $\mathcal{T}: \mathcal{K} \rightarrow C B(\mathcal{K})$ be a multivalued mapping. If $\mathcal{T}$ is a generalized $\alpha$-nonexpansive mapping, then the following inequality holds:

$$
d(x, \mathcal{T} y) \leq\left(\frac{3+\alpha}{1-\alpha}\right) d(x, \mathcal{T} x)+\|x-y\|, \quad \forall x, y \in \mathcal{K} .
$$

Lemma 2 [37]. Let $\left\{\theta_{n}\right\}$ be a nonnegative real sequence satisfying the following inequality:

$$
\theta_{n+1} \leq\left(1-\sigma_{n}\right) \theta_{n}
$$

where $\sigma_{n} \in(0,1)$ for all $n \in \mathbb{N}$ and

$$
\sum_{n=0}^{\infty} \sigma_{n}=\infty
$$

then

$$
\lim _{n \rightarrow \infty} \theta_{n}=0 .
$$

Lemma 3 [30]. Suppose $\mathscr{G}$ is a uniformly convex Banach space and $\left\{\iota_{n}\right\}$ is any sequence satisfying $0<p \leq \iota_{n} \leq q<1$ for all $n \geq 1$. Suppose $\left\{x_{n}\right\}$ and $\left\{y_{n}\right\}$ are any sequences of $\mathscr{G}$ such that

$$
\limsup _{n \rightarrow \infty}\left\|x_{n}\right\| \leq b, \quad \limsup _{n \rightarrow \infty}\left\|y_{n}\right\| \leq b
$$

and

$$
\limsup _{n \rightarrow \infty}\left\|\iota_{n} x_{n}+\left(1-\iota_{n}\right) y_{n}\right\|=b
$$

hold for some $b \geq 0$. Then $\lim _{n \rightarrow \infty}\left\|x_{n}-y_{n}\right\|=0$.

Lemma 4 [32]. Let $\mathcal{T}: \mathcal{K} \rightarrow \mathcal{P}(\mathcal{K})$ and

$$
\mathcal{P}_{\mathcal{T}}(x)=\{y \in \mathcal{T} x:\|x-y\|=d(x, \mathcal{T} x)\} .
$$

Then the following are equivalent

(a) $x \in F(\mathcal{T})$;

(b) $\mathcal{P}_{\mathcal{T}}(x)=\{x\}$;

(c) $x \in F\left(\mathcal{P}_{\mathcal{T}}\right)$.

Moreover, $F(\mathcal{T})=F\left(\mathcal{P}_{\mathcal{T}}\right)$. 


\section{Stability result}

In this section, we will show that the iterative algorithm $(1.9)$ is $w^{2}$-stable with respect to $\mathcal{T}$.

Theorem 1. Let $\mathcal{K}$ be a nonempty closed convex subset of a Banach space $\mathscr{G}$. Let $\mathcal{T}: \mathcal{K} \rightarrow \mathcal{P}(\mathcal{K})$ be a multivalued mapping and $\mathcal{P}_{\mathcal{T}}$ is a multivalued contraction mapping with $[0,1)$. Let $\left\{x_{n}\right\}$ be the iterative algorithm defined by (1.9), then $\left\{x_{n}\right\}$ converges to a fixed point of $\mathcal{T}$.

P r o o f. In [21], the existence of the fixed point of $\mathcal{P}_{\mathcal{T}}$ is guaranteed. Now, we show that $\left\{x_{n}\right\}$ converges to some fixed point $q$. Using (1.9), we have

$$
\begin{aligned}
& \left\|s_{n}-q\right\|=\left\|\left(1-v_{n}\right) x_{n}+v_{n} \ell_{n}-q\right\| \\
& \leq\left(1-v_{n}\right)\left\|x_{n}-q\right\|+v_{n}\left\|\ell_{n}-q\right\| \\
& \leq\left(1-v_{n}\right)\left\|x_{n}-q\right\|+v_{n} d\left(\ell_{n}, \mathcal{P}_{\mathcal{T}}(q)\right) \\
& \leq\left(1-v_{n}\right)\left\|x_{n}-q\right\|+v_{n} \mathscr{H}\left(\mathcal{P}_{\mathcal{T}}\left(x_{n}\right), \mathcal{P}_{\mathcal{T}}(q)\right) \\
& \leq\left(1-v_{n}\right)\left\|x_{n}-q\right\|+v_{n} \delta\left\|x_{n}-q\right\| \\
& =\left(1-v_{n}(1-\delta)\right)\left\|x_{n}-q\right\| \text {, } \\
& \left\|z_{n}-q\right\|=\left\|\left(1-u_{n}\right) \ell_{n}+u_{n} h_{n}-q\right\| \\
& \leq\left(1-u_{n}\right)\left\|\ell_{n}-q\right\|+u_{n}\left\|h_{n}-q\right\| \\
& \leq\left(1-u_{n}\right) d\left(\ell_{n}, \mathcal{P}_{\mathcal{T}}(q)\right)+u_{n} d\left(h_{n}, \mathcal{P}_{\mathcal{T}}(q)\right) \\
& \leq\left(1-u_{n}\right) \mathscr{H}\left(\mathcal{P}_{\mathcal{T}}\left(x_{n}\right), \mathcal{P}_{\mathcal{T}}(q)\right)+u_{n} \mathscr{H}\left(\mathcal{P}_{\mathcal{T}}\left(s_{n}\right), \mathcal{P}_{\mathcal{T}}(q)\right) \\
& \leq\left(1-u_{n}\right) \delta\left\|x_{n}-q\right\|+u_{n} \delta\left\|s_{n}-q\right\| \\
& \leq \delta\left(1-u_{n} v_{n}(1-\delta)\right)\left\|x_{n}-q\right\| \text {, } \\
& \left\|y_{n}-q\right\| \leq \mathscr{H}\left(\mathcal{P}_{\mathcal{T}}\left(z_{n}\right), \mathcal{P}_{\mathcal{T}}(q)\right) \\
& \leq \delta\left\|z_{n}-q\right\| \\
& \leq \delta^{2}\left(1-u_{n} v_{n}(1-\delta)\right)\left\|x_{n}-q\right\|, \\
& \left\|x_{n+1}-q\right\|=\left\|\zeta_{n}-q\right\| \\
& \leq \mathscr{H}\left(\mathcal{P}_{\mathcal{T}}\left(y_{n}\right), \mathcal{P}_{\mathcal{T}}(q)\right) \\
& \leq \delta\left\|y_{n}-q\right\| \\
& \leq \delta^{3}\left(1-u_{n} v_{n}(1-\delta)\right)\left\|x_{n}-q\right\| \text {. }
\end{aligned}
$$

Since $\left\{u_{n}\right\},\left\{v_{n}\right\} \in(0,1)$ and $\delta \in[0,1)$, it implies that

$$
\left(1-u_{n} v_{n}(1-\delta)\right)<1 .
$$

Thus, (3.1) yields

$$
\begin{aligned}
\left\|x_{n+1}-q\right\| & \leq \delta^{3}\left\|x_{n}-q\right\| \\
& \vdots \\
& \leq \gamma^{3 n}\left\|x_{1}-q\right\| .
\end{aligned}
$$

Taking limit on both sides of the above inequality (3.2), we get $\lim _{n \rightarrow \infty}\left\|x_{n}-q\right\|=0$. Indeed, $\delta \in[0,1)$ and so $\lim _{n \rightarrow \infty} \gamma^{3 n}=0$. 
We provide the following example to support our analytical proof in Theorem 1.

Example 1. Let $\mathcal{K}=[0,1] \subset \mathscr{G}=\mathbb{R}$ be endowed with the usual norm. Define an operator $\mathcal{T}: \mathcal{K} \rightarrow \mathcal{P}(\mathcal{K})$ by

$$
\mathcal{T} x=\left[0, \frac{x}{4}\right] .
$$

Clearly, $q=0 \in \mathcal{T} x$. Next we show that $\mathcal{P}_{\mathcal{T}}$ is a multivalued contraction mapping with $\delta=1 / 4$. Now

$$
\begin{aligned}
\mathcal{P}_{\mathcal{T}} & =\left\{y \in \mathcal{T}_{x}:|x-y|=d\left(x,\left[0, \frac{x}{4}\right]\right)\right\} \\
& =\left\{y \in \mathcal{T}_{x}:|x-y|=\left|x-\frac{x}{4}\right|\right\} \\
& =\left\{y \in \mathcal{T}_{x}: x-y=x-\frac{x}{4}\right\} \\
& =\left\{y \in \mathcal{T}_{x}: y=\frac{x}{4}\right\},
\end{aligned}
$$

so that

$$
\mathscr{H}(\mathcal{T} x, \mathcal{T} y) \leq \frac{1}{4}\|x-y\|
$$

for all $x, y \in \mathcal{T}$.

The iteration algorithm (1.9) associated with the mapping in (3.3) is as follows:

$$
\left\{\begin{array}{l}
x_{1} \in \mathcal{K}, \\
s_{n}=\left(1-v_{n}\right) x_{n}+v_{n} \frac{x_{n}}{4}, \\
z_{n}=\left(1-u_{n}\right) \frac{x_{n}}{4}+u_{n} \frac{s_{n}}{4}, \quad \forall n \geq 1 . \\
y_{n}=\frac{s_{n}}{4} \\
x_{n+1}=\frac{y_{n}}{4}
\end{array}\right.
$$

The following Table 1 and Fig. 1 show that $\lim _{n \rightarrow \infty}=0=q \in \mathcal{T} x$ for different choices of real sequences $\left\{u_{n}\right\}$ and $\left\{v_{n}\right\}$ in $(0,1)$.

Table 1. Convergence behavior of iteration algorithm (3.3) for different choices of real sequences $\left\{u_{n}\right\}$ and $\left\{v_{n}\right\}$ in $(0,1)$.

\begin{tabular}{|c|c|c|c|}
\hline Step & $(3.3)$ with $a$ & $(3.3)$ with $b$ & $(3.3)$ with $c$ \\
\hline 1 & 0.9000000000 & 0.9000000000 & 0.9000000000 \\
2 & 0.0114257812 & 0.0128906250 & 0.0123046875 \\
3 & 0.0001450539 & 0.0001846313 & 0.0001682281 \\
4 & 0.0000018415 & 0.0000026445 & 0.0000023000 \\
5 & 0.0000000234 & 0.0000000379 & 0.0000000314 \\
6 & 0.0000000003 & 0.0000000005 & 0.0000000004 \\
7 & 0.0000000000 & 0.0000000000 & 0.0000000000 \\
\hline
\end{tabular}

where $a, b$ and $c$ stand for the cases

$$
u_{n}=v_{n}=\frac{1}{n+1}, \quad u_{n}=v_{n}=\frac{1}{2 n+1}, \quad u_{n}=\frac{n+1}{2 n+1}, \quad v_{n}=\frac{n}{n^{2}+1},
$$


respectively.

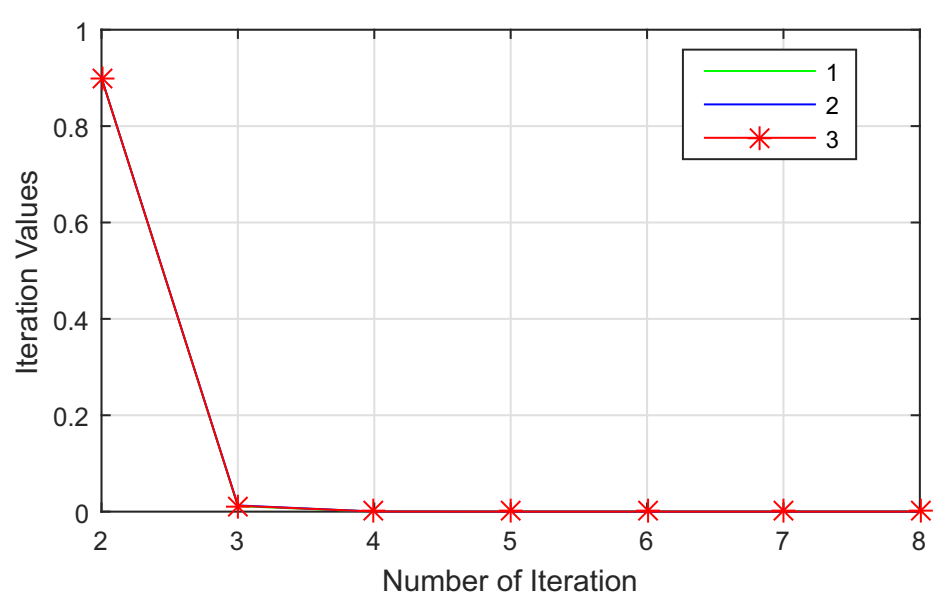

Figure 1. Graph corresponding to Table 1.

Theorem 2. Suppose that all the conditions of Theorem 1 are satisfied. Then the iteration algorithm (1.9) is $w^{2}$-stable with respect to $\mathcal{T}$.

P r o o f. Let $\left\{t_{n}\right\} \in \mathcal{K}$ be an equivalent sequence of $\left\{x_{n}\right\}$. Define a sequence $\left\{\epsilon_{n}\right\}$ in $\mathbb{R}^{+}$by

$$
\left\{\begin{array}{l}
\epsilon_{n}=\left\|t_{n+1}-m_{n}\right\|, \\
k_{n} \in \mathcal{P}_{\mathcal{T}}\left(g_{n}\right), \\
g_{n}=\left(1-u_{n}\right) r_{n}+u_{n} d_{n}, \quad \forall n \in \mathbb{N}, \\
c_{n}=\left(1-v_{n}\right) t_{n}+v_{n} r_{n},
\end{array}\right.
$$

where $\left\{u_{n}\right\}$ and $\left\{v_{n}\right\}$ are sequences in $(0,1), m_{n} \in \mathcal{P}_{\mathcal{T}}\left(k_{n}\right), k_{n} \in \mathcal{P}_{\mathcal{T}}\left(g_{n}\right), r_{n} \in \mathcal{P}_{\mathcal{T}}\left(x_{n}\right)$ and $d_{n} \in \mathcal{P}_{\mathcal{T}}\left(c_{n}\right)$. Let $\lim _{n \rightarrow \infty} \epsilon_{n}=0$, then from (1.1), (1.9) and (3.5), we have

$$
\begin{aligned}
&\left\|t_{n+1}-q\right\| \leq\left\|t_{n+1}-x_{n+1}\right\|+\left\|x_{n+1}-q\right\| \\
& \leq\left\|t_{n+1}-m_{n}\right\|+\left\|m_{n}-x_{n+1}\right\|+\left\|x_{n+1}-q\right\| \\
&=\epsilon_{n}+\left\|m_{n}-\zeta_{n}\right\|+\left\|x_{n+1}-q\right\| \\
& \leq \epsilon_{n}+\mathscr{H}\left(\mathcal{P}_{\mathcal{T}}\left(k_{n}\right), \mathcal{P}_{\mathcal{T}}\left(y_{n}\right)\right)+\left\|x_{n+1}-q\right\| \\
& \leq \epsilon_{n}+\delta\left\|k_{n}-y_{n}\right\|+\left\|x_{n+1}-q\right\| \\
& \leq \epsilon_{n}+\delta \mathscr{H}\left(\mathcal{P}_{\mathcal{T}}\left(g_{n}\right), \mathcal{P}_{\mathcal{T}}\left(z_{n}\right)\right)+\left\|x_{n+1}-q\right\| \\
& \leq \epsilon_{n}+\delta^{2}\left\|g_{n}-z_{n}\right\|+\left\|x_{n+1}-q\right\|, \\
&\left\|g_{n}-z_{n}\right\| \leq\left(1-u_{n}\right)\left\|r_{n}-\ell_{n}\right\|+u_{n}\left\|d_{n}-h_{n}\right\| \\
& \leq\left(1-u_{n}\right) \mathscr{H}\left(\mathcal{P}_{\mathcal{T}}\left(t_{n}\right), \mathcal{P}_{\mathcal{T}}\left(x_{n}\right)\right)+u_{n} \mathscr{H}\left(\mathcal{P}_{\mathcal{T}}\left(c_{n}\right), \mathcal{P}_{\mathcal{T}}\left(s_{n}\right)\right) \\
& \leq\left(1-u_{n}\right) \delta\left\|t_{n}-x_{n}\right\|+u_{n} \delta\left\|c_{n}-s_{n}\right\|, \\
&\left\|c_{n}-s_{n}\right\| \leq\left(1-v_{n}\right)\left\|t_{n}-x_{n}\right\|+u_{n}\left\|r_{n}-l_{n}\right\| \\
& \leq\left(1-v_{n}\right)\left\|t_{n}-x_{n}\right\|+u_{n} \mathscr{H}\left(\mathcal{P}_{\mathcal{T}}\left(t_{n}\right), \mathcal{P}_{\mathcal{T}}\left(x_{n}\right)\right) \\
& \leq\left(1-v_{n}\right)\left\|t_{n}-x_{n}\right\|+v_{n} \delta\left\|t_{n}-x_{n}\right\| \\
&=\left(1-v_{n}(1-\delta)\right)\left\|t_{n}-x_{n}\right\| .
\end{aligned}
$$


Using (3.6), (3.7) and (3.8), we get

$$
\left\|t_{n+1}-q\right\| \leq \epsilon_{n}+\delta^{3}\left(1-u_{n} v_{n}(1-\delta)\right)\left\|t_{n}-x_{n}\right\|+\left\|x_{n+1}-q\right\| .
$$

Since $\left\{t_{n}\right\} \in \mathcal{K}$ and its equivalence to $\left\{x_{n}\right\}$ yields $\lim _{n \rightarrow \infty}\left\|x_{n}-t_{n}\right\|=0$. We have shown in Theorem 1 that $\lim _{n \rightarrow \infty}\left\|x_{n}-q\right\|=0$, consequently $\lim _{n \rightarrow \infty}\left\|x_{n+1}-q\right\|=0$.

Thus, taking the limit on both sides of (3.9), we get

$$
\lim _{n \rightarrow \infty}\left\|t_{n}-q\right\|=0
$$

Hence, $\left\{x_{n}\right\}$ is $w^{2}$-stable with respect to $\mathcal{T}$.

We again support the analytical proof in Theorem 2 with the following example.

Example 2. Let $\mathcal{K}, \mathscr{G}=\mathbb{R}$ and $\mathcal{T}$ be same as in Example 1. Let $\left\{x_{n}^{(1)}\right\}_{n=0}^{\infty},\left\{x_{n}^{(2)}\right\}_{n=0}^{\infty}$ and $\left\{x_{n}^{(3)}\right\}_{n=0}^{\infty}$ be iterative algorithms corresponding to (3.4) with control parameters

$$
\left(u_{n}=v_{n}=\frac{1}{n+1}\right), \quad\left(u_{n}=v_{n}=\frac{1}{2 n+1}\right), \quad\left(u_{n}=\frac{n+1}{2 n+1}, \quad v_{n}=\frac{n}{n^{2}+1}\right)
$$

for all $n \in \mathbb{N}$, respectively.

It is shown in Example 1 that $\left\{x_{n}^{(i)}\right\}_{n=0}^{\infty}$ converges to $q=0 \in \mathcal{T} x$ for each $i \in\{1,2,3\}$. Clearly,

$$
\lim _{n \rightarrow \infty}\left\|x^{(i)}\right\|=\left\|\lim _{n \rightarrow \infty} x^{(i)}\right\|=0
$$

for each $i \in\{1,2,3\}$. Taking the sequence $\left\{t_{n}\right\}_{n=0}^{\infty}$ to be $t_{n}=1 /(n+4)$ for all $n \in \mathbb{N}$, then we get

$$
0 \leq \lim _{n \rightarrow \infty}\left\|x^{(i)}-t_{n}\right\| \leq \lim _{n \rightarrow \infty}\left\|x^{(i)}\right\|+\lim _{n \rightarrow \infty}\left\|t_{n}\right\|=0, \quad \text { for each } \quad i \in\{1,2,3\},
$$

which shows that $\lim _{n \rightarrow \infty}\left\|x^{(i)}-t_{n}\right\|=0$ for each $i \in\{1,2,3\}$, in other words, each of $\left\{x_{n}^{(i)}\right\}_{n=0}^{\infty}$, $i \in\{1,2,3\}$ and

$$
\left\{t_{n}\right\}_{n=0}^{\infty}=\left\{\frac{1}{n+4}\right\}_{n=0}^{\infty}
$$

are equivalent sequences.

Let $\epsilon_{n}^{(1)}, \epsilon_{n}^{(2)}$ and $\epsilon_{n}^{(3)}$ be corresponding sequences to the iterative algorithms $\left\{x_{n}^{(1)}\right\}_{n=0}^{\infty}$, $\left\{x_{n}^{(2)}\right\}_{n=0}^{\infty}$ and $\left\{x_{n}^{(3)}\right\}_{n=0}^{\infty}$, respectively. Then we have

$$
\begin{aligned}
& \epsilon_{n}^{(1)}=\left|\frac{1}{n+5}-\frac{1}{4}\left(\frac{1}{4}\left(\frac{n}{n+1} \cdot \frac{1}{4} \cdot \frac{1}{n+4}+\frac{1}{n+1} \cdot \frac{1}{4}\left(\frac{n}{n+1} \cdot \frac{1}{n+4}+\frac{1}{n+1} \cdot \frac{1}{4} \cdot \frac{1}{n+4}\right)\right)\right)\right|, \\
& \epsilon_{n}^{(2)}=\left|\frac{1}{n+5}-\frac{1}{4}\left(\frac{1}{4}\left(\frac{2 n}{2 n+1} \cdot \frac{1}{4} \cdot \frac{1}{n+4}+\frac{1}{2 n+1} \cdot \frac{1}{4}\left(\frac{2 n}{2 n+1} \cdot \frac{1}{n+4}+\frac{1}{2 n+1} \cdot \frac{1}{4} \cdot \frac{1}{n+4}\right)\right)\right)\right|,
\end{aligned}
$$

and

$$
\epsilon_{n}^{(3)}=\left|\frac{1}{n+5}-\frac{1}{4}\left(\frac{1}{4}\left(\frac{n^{2}-n+1}{n^{2}+1} \cdot \frac{1}{4} \cdot \frac{1}{n+4}+\frac{n}{n^{2}+1} \cdot \frac{1}{4}\left(\frac{n}{2 n+1} \cdot \frac{1}{n+4}+\frac{n+1}{2 n+1} \cdot \frac{1}{4} \cdot \frac{1}{n+4}\right)\right)\right)\right| .
$$

Obviously, $\lim _{n \rightarrow \infty} \epsilon_{n}^{(i)}=0$ for each $i \in\{1,2,3\}$. Hence, all the iterative algorithms $\left\{x_{n}^{(i)}\right\}_{n=0}^{\infty}$, $i \in\{1,2,3\}$ are $w^{2}$-stable with respect to $\mathcal{T}$.

Remark 1. Since the notion of $w^{2}$-stability is more general the concept of simple stability considered in [15, 24, 28], hence, our result improves and generalizes the corresponding in $[15,24,28]$ and several others. 


\section{Convergence results}

In this section, we will prove the weak and strong convergence results of our new iterative algorithm (1.9) for multivalued generalized $\alpha$-nonexpansive mappings in uniformly convex Banach spaces.

Lemma 5. Let $\mathcal{K}$ be a nonempty closed convex subset of a real Banach space $\mathscr{G}$. Let $\mathcal{T}: \mathcal{K} \rightarrow \mathcal{P}(\mathcal{K})$ be a multivalued mapping such that $F(\mathcal{T}) \neq \emptyset$ and $\mathcal{P}_{\mathcal{T}}$ is a generalized $\alpha$-nonexpansive mapping. Let $\left\{x_{n}\right\}$ be the iterative algorithm defined by (1.9), then $\lim _{n \rightarrow \infty}\left\|x_{n}-q\right\|$ exists for all $q \in F(\mathcal{T})$.

P r o o f. Taking $q \in F(\mathcal{T})$, then from Lemma 4 , we have $\mathcal{P}_{\mathcal{T}}(q)=\{q\}$ and $\mathcal{P}(\mathcal{T})=F\left(\mathcal{P}_{\mathcal{T}}\right)$. Since $\mathcal{P}_{\mathcal{T}}$ is a generalized $\alpha$-nonexpansive mapping, we get

$$
\frac{1}{2} d\left(q, \mathcal{P}_{\mathcal{T}}(q)\right)=0=\left\|x_{n}-q\right\| .
$$

On the other hand,

$$
\begin{aligned}
\mathscr{H}\left(\mathcal{P}_{\mathcal{T}}\left(x_{n}\right), \mathcal{P}_{\mathcal{T}}(q)\right) & \leq \alpha d\left(x_{n}, \mathcal{P}_{\mathcal{T}}(q)\right)+\alpha d\left(q, \mathcal{P}_{\mathcal{T}}\left(x_{n}\right)\right)+(1-2 \alpha)\left\|x_{n}-q\right\| \\
& \leq \alpha\left\|x_{n}-q\right\|+\alpha \mathscr{H}\left(\mathcal{P}_{\mathcal{T}}(q), \mathcal{P}_{\mathcal{T}}\left(x_{n}\right)\right)+(1-2 \alpha)\left\|x_{n}-q\right\| \\
& \leq\left\|x_{n}-q\right\| .
\end{aligned}
$$

Similarly, for any $q \in F(\mathcal{T})$, we obtain

$$
\left\{\begin{array}{l}
\mathscr{H}\left(\mathcal{P}_{\mathcal{T}}\left(y_{n}\right), \mathcal{P}_{\mathcal{T}}(q)\right) \leq\left\|y_{n}-q\right\|, \\
\mathscr{H}\left(\mathcal{P}_{\mathcal{T}}\left(z_{n}\right), \mathcal{P}_{\mathcal{T}}(q)\right) \leq\left\|z_{n}-q\right\|, \\
\mathscr{H}\left(\mathcal{P}_{\mathcal{T}}\left(s_{n}\right), \mathcal{P}_{\mathcal{T}}(q)\right) \leq\left\|s_{n}-q\right\|, \\
\mathscr{H}\left(\mathcal{P}_{\mathcal{T}}\left(\zeta_{n}\right), \mathcal{P}_{\mathcal{T}}(q)\right) \leq\left\|\zeta_{n}-q\right\| .
\end{array}\right.
$$

Now from (1.9), we have

$$
\begin{aligned}
\left\|s_{n}-q\right\| & =\left\|\left(1-v_{n}\right) x_{n}+v_{n} \ell_{n}-q\right\| \\
& \leq\left(1-v_{n}\right)\left\|x_{n}-q\right\|+v_{n}\left\|\ell_{n}-q\right\| \\
& \leq\left(1-v_{n}\right)\left\|x_{n}-q\right\|+v_{n} d\left(\ell_{n}, \mathcal{P}_{\mathcal{T}}(q)\right) \\
& \leq\left(1-v_{n}\right)\left\|x_{n}-q\right\|+v_{n} \mathscr{H}\left(\mathcal{P}_{\mathcal{T}}\left(x_{n}\right), \mathcal{P}_{\mathcal{T}}(q)\right) \\
& \leq\left(1-v_{n}\right)\left\|x_{n}-q\right\|+v_{n}\left\|x_{n}-q\right\| \\
& =\left\|x_{n}-q\right\| .
\end{aligned}
$$

Also,

$$
\begin{aligned}
\left\|z_{n}-q\right\| & =\left\|\left(1-u_{n}\right) \ell_{n}+u_{n} h_{n}-q\right\| \\
& \leq\left(1-u_{n}\right)\left\|\ell_{n}-q\right\|+u_{n}\left\|h_{n}-q\right\| \\
& \leq\left(1-u_{n}\right) d\left(\ell_{n}, \mathcal{P}_{\mathcal{T}}(q)\right)+u_{n} d\left(h_{n}, \mathcal{P}_{\mathcal{T}}(q)\right) \\
& \leq\left(1-u_{n}\right) \mathscr{H}\left(\mathcal{P}_{\mathcal{T}}\left(x_{n}\right), \mathcal{P}_{\mathcal{T}}(q)\right)+u_{n} \mathscr{H}\left(\mathcal{P}_{\mathcal{T}}\left(s_{n}\right), \mathcal{P}_{\mathcal{T}}(q)\right) \\
& \leq\left(1-u_{n}\right)\left\|x_{n}-q\right\|+u_{n}\left\|s_{n}-q\right\| \\
& \leq\left\|x_{n}-q\right\| .
\end{aligned}
$$

Again,

$$
\begin{aligned}
\left\|y_{n}-q\right\| & \leq \mathscr{H}\left(\mathcal{P}_{\mathcal{T}}\left(z_{n}\right), \mathcal{P}_{\mathcal{T}}(q)\right) \\
& \leq\left\|z_{n}-q\right\| \\
& \leq\left\|x_{n}-q\right\| .
\end{aligned}
$$


Finally,

$$
\begin{aligned}
\left\|x_{n+1}-q\right\| & =\left\|\zeta_{n}-q\right\| \\
& \leq \mathscr{H}\left(\mathcal{P}_{\mathcal{T}}\left(y_{n}\right), \mathcal{P}_{\mathcal{T}}(q)\right) \\
& \leq\left\|y_{n}-q\right\| \\
& \leq\left\|x_{n}-q\right\| .
\end{aligned}
$$

Thus, $\left\{\left\|x_{n}-q\right\|\right\}$ is bounded and non-increasing, which implies that $\lim _{n \rightarrow \infty}\left\|x_{n}-q\right\|$ exists for all $q \in F(\mathcal{T})$.

Lemma 6. Let $\mathcal{K}$ be a nonempty subset of a uniformly convex Banach space $\mathscr{G}$. Let $\mathcal{T}: \mathcal{K} \rightarrow \mathcal{P}(\mathcal{K})$ be a multivalued mapping such that $F(\mathcal{T}) \neq \emptyset$ and $\mathcal{P}_{\mathcal{T}}$ is a generalized $\alpha$-nonexpansive mapping. Let $\left\{x_{n}\right\}$ be the iterative algorithm defined by (1.9), then $\lim _{n \rightarrow \infty} d\left(x_{n}, \mathcal{T} x_{n}\right)=0$.

P r o o f. From Lemma 5, we have that $\lim _{n \rightarrow \infty}\left\|x_{n}-q\right\|$ exists for all $q \in F(\mathcal{T})$. We suppose that

$$
\lim _{n \rightarrow \infty}\left\|x_{n}-q\right\|=b \quad \text { for some } \quad b \geq 0 .
$$

Now from (4.1), (4.2), (4.3) and (4.4), we have

$$
\begin{aligned}
& \limsup _{n \rightarrow \infty}\left\|s_{n}-q\right\| \leq b, \\
& \limsup _{n \rightarrow \infty}\left\|z_{n}-q\right\| \leq b, \\
& \limsup _{n \rightarrow \infty}\left\|y_{n}-q\right\| \leq b
\end{aligned}
$$

and

$$
\lim _{n \rightarrow \infty}\left\|\zeta_{n}-q\right\| \leq b
$$

Now, we have the following inequalities

$$
\begin{aligned}
\limsup _{n \rightarrow \infty}\left\|\ell_{n}-q\right\| & \leq \limsup _{n \rightarrow \infty} \mathscr{H}\left(\mathcal{P}_{\mathcal{T}}\left(x_{n}\right), \mathcal{P}_{\mathcal{T}}(q)\right) \\
& \leq \limsup _{n \rightarrow \infty}\left\|x_{n}-q\right\|=b, \\
\limsup _{n \rightarrow \infty}\left\|h_{n}-q\right\| & \leq \limsup _{n \rightarrow \infty} \mathscr{H}\left(\mathcal{P}_{\mathcal{T}}\left(s_{n}\right), \mathcal{P}_{\mathcal{T}}(q)\right) \\
& \leq \limsup _{n \rightarrow \infty}\left\|s_{n}-q\right\| \leq b
\end{aligned}
$$

and

$$
\begin{aligned}
\limsup _{n \rightarrow \infty}\left\|\zeta_{n}-q\right\| & \leq \limsup _{n \rightarrow \infty} \mathscr{H}\left(\mathcal{P}_{\mathcal{T}}\left(y_{n}\right), \mathcal{P}_{\mathcal{T}}(q)\right) \\
& \leq \limsup _{n \rightarrow \infty}\left\|y_{n}-q\right\| \leq b
\end{aligned}
$$

Using (1.9) and (4.4), we have

$$
\begin{aligned}
b=\lim _{n \rightarrow \infty}\left\|x_{n+1}-q\right\| & =\lim _{n \rightarrow \infty}\left\|\zeta_{n}-q\right\| \\
& \leq \lim _{n \rightarrow \infty} \mathscr{H}\left(\mathcal{P}_{\mathcal{T}}\left(y_{n}\right), \mathcal{P}_{\mathcal{T}}(q)\right) \\
& \leq \lim _{n \rightarrow \infty}\left\|y_{n}-q\right\| \\
& \leq \lim _{n \rightarrow \infty} \mathscr{H}\left(\mathcal{P}_{\mathcal{T}}\left(z_{n}\right), \mathcal{P}_{\mathcal{T}}(q)\right) \\
& \leq \lim _{n \rightarrow \infty}\left\|z_{n}-q\right\| \\
& =\lim _{n \rightarrow \infty}\left\|\left(1-u_{n}\right) \ell_{n}+u_{n} h_{n}-q\right\| .
\end{aligned}
$$


By Lemma 3, we have

$$
\lim _{n \rightarrow \infty}\left\|\ell_{n}-h_{n}\right\|=0 .
$$

Again, from (1.9) we have

$$
\begin{aligned}
\left\|x_{n+1}-q\right\| & =\left\|\zeta_{n}-q\right\| \\
& \leq \mathscr{H}\left(\mathcal{P}_{\mathcal{T}}\left(y_{n}\right), \mathcal{P}_{\mathcal{T}}(q)\right) \\
& \leq\left\|y_{n}-q\right\|,
\end{aligned}
$$

which gives

$$
b \leq \liminf _{n \rightarrow \infty}\left\|y_{n}-q\right\| .
$$

From (4.7) and (4.11), we obtain

$$
\lim _{n \rightarrow \infty}\left\|y_{n}-q\right\|=b
$$

Again from (1.9), we have

$$
\begin{aligned}
\left\|y_{n}-q\right\| & \leq \mathscr{H}\left(\mathcal{P}_{\mathcal{T}}\left(z_{n}\right), \mathcal{P}_{\mathcal{T}}(q)\right) \\
& \leq\left\|z_{n}-q\right\|,
\end{aligned}
$$

which yields

$$
b \leq \liminf _{n \rightarrow \infty}\left\|z_{n}-q\right\| .
$$

From (4.6) and (4.12), we have

$$
\lim _{n \rightarrow \infty}\left\|z_{n}-q\right\|=b .
$$

By (1.9) and (4.10), we get

$$
\begin{aligned}
\left\|z_{n}-q\right\| & =\left\|\left(1-u_{n}\right) \ell_{n}+u_{n} h_{n}-q\right\| \\
& \leq\left\|\ell_{n}-q\right\|+u_{n}\left\|h_{n}-\ell_{n}\right\|,
\end{aligned}
$$

which gives

$$
b \leq \liminf _{n \rightarrow \infty}\left\|\ell_{n}-q\right\| .
$$

Using (4.9) and (4.13), we have

$$
\lim _{n \rightarrow \infty}\left\|\ell_{n}-q\right\|=b .
$$

Also,

$$
\begin{aligned}
\left\|\ell_{n}-q\right\| & \leq\left\|\ell_{n}-h_{n}\right\|+\left\|h_{n}-q\right\| \\
& \leq\left\|\ell_{n}-h_{n}\right\|+\mathscr{H}\left(\mathcal{P}_{\mathcal{T}}\left(s_{n}\right), \mathcal{P}_{\mathcal{T}}(q)\right) \\
& \leq\left\|\ell_{n}-h_{n}\right\|+\left\|h_{n}-q\right\|
\end{aligned}
$$

gives

$$
b \leq \liminf _{n \rightarrow \infty}\left\|s_{n}-q\right\| .
$$


From (4.5) and (4.14), we obtain

$$
\lim _{n \rightarrow \infty}\left\|s_{n}-q\right\|=b
$$

Finally, from (1.9), we have

$$
\lim _{n \rightarrow \infty}\left\|s_{n}-q\right\|=\lim _{n \rightarrow \infty}\left\|\left(1-v_{n}\right)\left(x_{n}-q\right)+v_{n}\left(\ell_{n}-q\right)\right\|=b .
$$

Now, due to (4.4), (4.9), (4.15) and Lemma 3 we obtain

$$
\lim _{n \rightarrow \infty}\left\|x_{n}-\ell_{n}\right\|=0 .
$$

Since $d\left(x_{n}, \mathcal{T} x_{n}\right) \leq\left\|x_{n}-\ell_{n}\right\|$, we get

$$
\lim _{n \rightarrow \infty} d\left(x_{n}, \mathcal{T} x_{n}\right)=0 .
$$

Next we prove weak convergence of the iterative algorithm (1.9) to the fixed point of multivalued generalized $\alpha$-nonexpansive mapping.

Theorem 3. Let $\mathcal{K}$ be a nonempty subset of a uniformly convex Banach space $\mathscr{G}$ which satisfies Opial's condition. Let $\mathcal{T}: \mathcal{K} \rightarrow \mathcal{P}(\mathcal{K})$ be a multivalued mapping such that $F(\mathcal{T}) \neq \emptyset$ and $\mathcal{P}_{\mathcal{T}}$ is a generalized $\alpha$-nonexpansive mapping. Let $I-\mathcal{P}_{\mathcal{T}}$ be demiclosed with respect to zero and $\left\{x_{n}\right\}$ be the iterative algorithm defined by (1.9), then $\left\{x_{n}\right\}$ converges weekly to a fixed point of $\mathcal{T}$.

P r o o f. Let $q \in F(\mathcal{T})=F\left(\mathcal{P}_{\mathcal{T}}\right)$. From Lemma 5 we have that $\lim _{n \rightarrow \infty}\left\|x_{n}-q\right\|$ exists. Now we show that the sequence $\left\{x_{n}\right\}$ has a unique weak sequential limit in $F(\mathcal{T})$. To prove this, let $p_{1}$ and $p_{2}$ be weak limits of the subsequences $\left\{x_{n_{i}}\right\}$ and $\left\{x_{n_{k}}\right\}$ of $\left\{x_{n}\right\}$, respectively. From (4.16), there exists $\ell_{n} \in \mathcal{T} x_{n}$ such that $\lim _{n \rightarrow \infty}\left\|x_{n}-\ell_{n}\right\|=0$. Therefore, from the demiclosedness of $I-\mathcal{P}_{\mathcal{T}}$ with respect to zero, we have $p_{1} \in F(\mathcal{T})=F\left(\mathcal{P}_{\mathcal{T}}\right)$. Following the same method of proof, we can show that $p_{2} \in F(\mathcal{T})$. Next, we prove uniqueness. To show this, suppose that $p_{1} \neq p_{2}$, then from Opial's condition we obtain

$$
\begin{aligned}
\lim _{n \rightarrow \infty}\left\|x_{n}-p_{1}\right\| & =\lim _{n_{i} \rightarrow \infty}\left\|x_{n_{i}}-p_{1}\right\| \\
& <\lim _{n_{i} \rightarrow \infty}\left\|x_{n_{i}}-p_{2}\right\| \\
& =\lim _{n \rightarrow \infty}\left\|x_{n}-p_{2}\right\| \\
& =\lim _{n_{k} \rightarrow \infty}\left\|x_{n_{k}}-p_{2}\right\| \\
& <\lim _{n_{k} \rightarrow \infty}\left\|x_{n_{k}}-p_{1}\right\| \\
& =\lim _{n \rightarrow \infty}\left\|x_{n}-p_{1}\right\|,
\end{aligned}
$$

which is a contradiction, so $p_{1}=p_{2}$. Hence, $\left\{x_{n}\right\}$ converges weakly to a fixed point of $\mathcal{T}$.

Furthermore, we state and prove strong convergence theorems of the new iterative algorithm (1.9) for multivalued generalized $\alpha$-nonexpansive mappings.

Theorem 4. Let $\mathcal{K}$ be a nonempty closed convex subset of a real Banach space $\mathscr{G}$. Let $\mathcal{T}$ : $\mathcal{K} \rightarrow \mathcal{P}(\mathcal{K})$ be a multivalued mapping such that $F(\mathcal{T}) \neq \emptyset$ and $\mathcal{P}_{\mathcal{T}}$ is a generalized $\alpha$-nonexpansive mapping. If $\left\{x_{n}\right\}$ be the iterative algorithm defined by (1.9), then $\left\{x_{n}\right\}$ converges strongly to a fixed point of $\mathcal{T}$ if and only if $\liminf _{n \rightarrow \infty} d\left(x_{n}, F(\mathcal{T})\right)=0$. 
P r o o f. The necessity is obvious. Conversely, assume that $\liminf _{n \rightarrow \infty} d\left(x_{n}, F(\mathcal{T})\right)=0$. By Lemma 5 , it is proved that

$$
\left\|x_{n+1}-q\right\| \leq\left\|x_{n}-q\right\|
$$

This yields

$$
d\left(x_{n+1}, F(\mathcal{T})\right) \leq d\left(x_{n}, F(\mathcal{T})\right) .
$$

Thus $\liminf _{n \rightarrow \infty} d\left(x_{n}, F(\mathcal{T})\right)$ exists. By hypothesis,

$$
\liminf _{n \rightarrow \infty} d\left(x_{n}, F(\mathcal{T})\right)=0
$$

so we must have

$$
\lim _{n \rightarrow \infty} d\left(x_{n}, F(\mathcal{T})\right)=0 .
$$

Next, we prove that the sequence $\left\{x_{n}\right\}$ is Cauchy in $\mathcal{K}$. We choose arbitrary $\epsilon>0$. Since $\lim _{n \rightarrow \infty} d\left(x_{n}, F(\mathcal{T})\right)=0$, then there exists $n_{0}$ such that for all $n \geq n_{0}$.

$$
d\left(x_{n}, F(\mathcal{T})\right)<\frac{\epsilon}{4}
$$

Particularly,

$$
\inf \left\{\left\|x_{n_{0}}-q\right\|: q \in F(\mathcal{T})\right\}<\frac{\epsilon}{4}
$$

so an element $p \in F(\mathcal{T})$ must exist such that

$$
\left\|x_{n_{0}}-q\right\|<\frac{\epsilon}{2}
$$

Now for $n, s \geq n_{0}$, we have

$$
\left\|x_{n+s}-x_{n}\right\| \leq\left\|x_{n+s}-p\right\|+\left\|x_{n}-p\right\| \leq 2\left\|x_{n_{0}}-p\right\|<2\left(\frac{\epsilon}{2}\right)=\epsilon .
$$

Hence, $\left\{x_{n}\right\}$ is the Cauchy sequence in the closed subset $\mathcal{K}$ of the Banach space $\mathscr{G}$. It follows that $\left\{x_{n}\right\}$ must converge in $\mathcal{K}$. Now let $\lim _{n \rightarrow \infty} x_{n}=p^{*}$, then from Lemma 1 we obtain

$$
\begin{aligned}
d\left(p^{*}, \mathcal{P}_{\mathcal{T}}\left(p^{*}\right)\right) & \leq\left\|x_{n}-p^{*}\right\|+d\left(x_{n}, \mathcal{P}_{\mathcal{T}}\left(p^{*}\right)\right) \\
& \leq\left\|x_{n}-p^{*}\right\|+\left(\frac{3+\alpha}{1-\alpha}\right) d\left(x_{n}, \mathcal{P}_{\mathcal{T}}\left(x_{n}\right)\right)+\left\|x_{n}-p^{*}\right\| \rightarrow 0 \quad \text { as } \quad n \rightarrow \infty .
\end{aligned}
$$

This implies that $p^{*} \in \mathcal{P}_{\mathcal{T}}\left(p^{*}\right)$ and $p^{*} \in F\left(\mathcal{P}_{\mathcal{T}}\right)$. From Lemma 4 , we have $p^{*} \in F\left(\mathcal{P}_{\mathcal{T}}\right)$. Hence, $\left\{x_{n}\right\}$ converges strongly to a fixed point of $\mathcal{T}$.

Theorem 5. Let $\mathcal{K}$ be a nonempty compact convex subset of a uniformly convex Banach space $\mathscr{G}$. Let $\mathcal{T}: \mathcal{K} \rightarrow \mathcal{P}(\mathcal{K})$ be a multivalued mapping such that $F(\mathcal{T}) \neq \emptyset$ and $\mathcal{P}_{\mathcal{T}}$ is a generalized $\alpha$-nonexpansive mapping. Suppose $\left\{x_{n}\right\}$ is the iterative algorithm defined by (1.9), then $\left\{x_{n}\right\}$ converges strongly to a fixed point of $\mathcal{T}$.

P r o o f. By Lemma 5, we know that $\left\{x_{n}\right\}$ is bounded and $\lim _{n \rightarrow \infty} d\left(x_{n}, \mathcal{T} x_{n}\right)=0$. Since $\mathcal{K}$ is compact, it follows that a subsequence $\left\{x_{n_{i}}\right\}$ of $\left\{x_{n}\right\}$ exists such that $x_{n_{i}}$ converges to some $y \in \mathcal{K}$. Since $\mathcal{P}_{\mathcal{T}}$ is a multivalued generalized $\alpha$ nonexpansive mappings, then from Lemma 1 we obtain

$$
d\left(x_{n_{i}}, \mathcal{P}_{\mathcal{T}}(y)\right) \leq\left(\frac{3+\alpha}{1-\alpha}\right) d\left(x_{n_{i}}, \mathcal{T}\left(x_{n_{i}}\right)+\left\|x_{n_{i}}-y\right\|\right.
$$

Again, since $F(\mathcal{T})=F\left(\mathcal{P}_{\mathcal{T}}\right)$, by taking the limit as $i \rightarrow \infty$, we have that $y \in \mathcal{T} y$. Hence, $\left\{x_{n}\right\}$ converges strongly to $y \in F(\mathcal{T})$. 
Theorem 6. Let $\mathcal{K}$ be a nonempty closed convex subset of a real Banach space $\mathscr{G}$. Let $\mathcal{T}: \mathcal{K} \rightarrow \mathcal{P}(\mathcal{K})$ be a multivalued mapping satisfying condition (I) such that $F(\mathcal{T}) \neq \emptyset$ and $\mathcal{P}_{\mathcal{T}}$ is a generalized $\alpha$-nonexpansive mapping. If $\left\{x_{n}\right\}$ be the iterative algorithm defined by (1.9), then $\left\{x_{n}\right\}$ converges strongly to a fixed point of $\mathcal{T}$.

P r o o f. From Lemma $5, \lim _{n \rightarrow \infty}\left\|x_{n}-q\right\|$ exits for all $q \in F(\mathcal{T})$ and therefore $\left\{x_{n}\right\}$ is bounded. Let $\lim _{n \rightarrow \infty}\left\|x_{n}-q\right\|=b$ for some $b \geq 0$. If $b=0$, then the result follows trivially. Suppose $b>0$, then by Lemma 5 we have

$$
\left\|x_{n+1}-q\right\| \leq\left\|x_{n}-q\right\|
$$

which gives

$$
\inf _{q \in F(\mathcal{T})}\left\|x_{n+1}-q\right\| \leq \inf _{q \in F(\mathcal{T})}\left\|x_{n}-q\right\|
$$

It follows that

$$
d\left(x_{n+1}, F(\mathcal{T})\right) \leq d\left(x_{n}, F(\mathcal{T})\right),
$$

so $\lim _{n \rightarrow \infty} d\left(x_{n}, F(\mathcal{T})\right)$ exists. From condition $(I)$ and Lemma 6, we get

$$
\lim _{n \rightarrow \infty} f\left(d\left(x_{n}, F(\mathcal{T})\right)\right) \leq \lim _{n \rightarrow \infty} d\left(x_{n}, \mathcal{T} x_{n}\right)=0 .
$$

Since $f$ is a nondecreasing function and $f(0)=0$, it follows that $\lim _{n \rightarrow \infty} d\left(x_{n}, F(\mathcal{T})\right)=0$. Conclusion of the result follows from Theorem 4 .

\section{Numerical Experiment}

In this section, we give an example of a multivalued generalized $\alpha$-nonexpansive mapping which does not satisfy condition $(C)$. We will also compare the convergence of our new iterative algorithm with the iterative algorithms (1.2)-(1.6) using the provided example.

Example 3. Let $(\mathbb{R},\|\cdot\|)$ be a normed space with the usual norm and $\mathcal{K}=[2,4]$. Define $\mathcal{T}: \mathcal{K} \rightarrow \mathcal{P}(\mathcal{K})$ as:

$$
\mathcal{T} x=\left\{\begin{array}{lll}
{\left[2, \frac{x+2}{2}\right],} & \text { if } & x \in[2,3], \\
2, & \text { if } & x \in(3,4] .
\end{array}\right.
$$

Then $\mathcal{T}$ is a multivalued generalized $\alpha$-nonexpansive mapping, but $\mathcal{T}$ does not satisfy condition $(C)$.

First, we show that $\mathcal{T}$ is a multivalued generalized $1 / 3$-nonexpansive mapping. For this, we consider the following possible cases:

Case (a): If $x, y \in[2,3]$, then

$$
\begin{aligned}
\alpha d(\mathcal{T} x, y)+\alpha d(\mathcal{T} y, x)+(1-2 \alpha)\|x-y\| & =\frac{1}{3}\left|\frac{x+2}{2}-y\right|+\frac{1}{3}\left|\frac{y+2}{2}-x\right|+\frac{1}{3}|x-y| \\
& \geq \frac{1}{3}\left|\frac{3 x}{2}-\frac{3 x}{2}\right|+\frac{1}{3}|x-y| \\
& =\frac{1}{2}|x-y|+\frac{1}{3}|x-y| \\
& \geq \frac{1}{2}|x-y| \\
& =\mathscr{H}(\mathcal{T} x, \mathfrak{T} y) .
\end{aligned}
$$


Case (b): If $x \in[2,3]$ and $y \in(3,4]$, we obtain

$$
\begin{aligned}
\alpha d(\mathcal{T} x, y)+\alpha d(\mathcal{T} y, x)+(1-2 \alpha)\|x-y\| & =\frac{1}{3}\left|\frac{x+2}{2}-y\right|+\frac{1}{3}|x-2|+\frac{1}{3}|x-y| \\
& \geq \frac{1}{3}\left|\frac{x}{2}+y-\frac{6}{2}\right|+\frac{1}{3}|x-y| \\
& \geq \frac{1}{3}\left|\frac{3 x}{2}-\frac{6}{2}\right|+\frac{1}{3}|x-y| \\
& =\frac{1}{2}|x-2| \\
& =\mathscr{H}(\mathcal{T} x, \mathcal{T} y) .
\end{aligned}
$$

Case (c): If $x, y \in(3,4]$, then we have

$$
d(\mathcal{T} y, x)+\alpha d(\mathcal{T} y, x)+(1-2 \alpha)\|x-y\| \geq 0=\mathscr{H}(\mathcal{T} x, \mathcal{T} y) .
$$

Hence, $\mathcal{T}$ is a multivalued generalized $1 / 3$-nonexpansive mapping.

Next we show that $\mathcal{T}$ does not satisfy condition $(C)$. Now, take $x=29 / 10$ and $y=19 / 6$, then we obtain

$$
\frac{1}{2} d(x, \mathcal{T} x)=\left(\frac{29}{10}, \mathcal{T} \frac{29}{10}\right)=\frac{9}{40}<\frac{16}{60}=|x-y| .
$$

But,

$$
\mathscr{H}(\mathcal{T} x, \mathcal{T} y)=\frac{9}{20}>\frac{16}{60}=|x-y| .
$$

Hence, $\mathcal{T}$ does not satisfy condition $(C)$.

Finally, we will now show that $\mathcal{P}_{\mathcal{T}}$ is a multivalued generalized $\alpha$-nonexpansive mapping. Note that $q=2 \in \mathcal{T} x$. We consider the following cases:

Case (I): If $x \in[2,3]$, then

$$
\begin{aligned}
\mathcal{P}_{\mathcal{T}} & =\left\{y \in \mathcal{T} x:|y-x|=d\left(x,\left[1, \frac{x+2}{2}\right]\right)\right\} \\
& =\left\{y \in \mathcal{T} x:|y-x|=\left|x-\frac{x+2}{2}\right|\right\} \\
& =\left\{y \in \mathcal{T} x:|y-x|=\left|\frac{x-2}{2}\right|\right\} \\
& =\left\{y \in \mathcal{T} x: x-y=\left|\frac{x-2}{2}\right|\right\} \\
& =\left\{y \in \mathcal{T}_{x}: y=\frac{x+2}{2}\right\} .
\end{aligned}
$$

Case (II): If $x \in(3,4]$, then we get

$$
\begin{aligned}
\mathcal{P}_{\mathcal{T}} & =\{y \in \mathcal{T} x:|y-x|=d(x,\{2\}\} \\
& =\{y \in \mathcal{T} x:|y-x|=|x-2|\} \\
& =\{y \in \mathcal{T} x: x-y=x-2\} \\
& =\{y \in \mathcal{T} x: y=2\} .
\end{aligned}
$$

Following the same argument as those in Example 3, one can easily show that $\mathcal{P}_{\mathcal{T}}$ is a multivalued generalized $\alpha$-nonexpansive mapping. 
With the aid of MATLAB (R2015a), we will use the above example to show that our new iterative algorithm (1.9) converges faster than the iterative algorithms (1.2)-(1.6) and the comparison Table 2 and Fig. 2 are obtained for various iterative algorithms with control sequences $u_{n}=v_{n}=t_{n}=3 / 4$ and initial guess $x_{0}=4$.

Table 2. Comparison of speed of convergence of our new iterative algorithm (1.9) with Mann, Ishikawa, S, Thakur, Picard-Ishikawa iterative schemes.

\begin{tabular}{|c|c|c|c|c|c|c|}
\hline Step & Mann & Ishikawa & S & Thakur & Picard-Ishikawa & New \\
\hline 1 & 4.0000000 & 4.0000000 & 4.0000000 & 4.0000000 & 4.0000000 & 4.0000000 \\
2 & 3.2500000 & 2.9687500 & 2.7187500 & 2.6054688 & 2.4843750 & 2.1796875 \\
3 & 2.7812500 & 2.4692383 & 2.2583008 & 2.1832962 & 2.1173096 & 2.0161438 \\
4 & 2.4882813 & 2.2272873 & 2.0928268 & 2.0554901 & 2.0284109 & 2.0014504 \\
5 & 2.3051758 & 2.1100923 & 2.0333596 & 2.0167987 & 2.0068808 & 2.0001303 \\
6 & 2.1907349 & 2.0533259 & 2.0119886 & 2.0050856 & 2.0016664 & 2.0000117 \\
7 & 2.1192093 & 2.0258298 & 2.0043084 & 2.0015396 & 2.0004036 & 2.0000011 \\
8 & 2.0745058 & 2.0125113 & 2.0015483 & 2.0004661 & 2.0000977 & 2.0000001 \\
9 & 2.0465661 & 2.0060602 & 2.0005564 & 2.0001411 & 2.0000237 & 2.0000000 \\
10 & 2.0291038 & 2.0029354 & 2.0002000 & 2.0000427 & 2.0000057 & 2.0000000 \\
11 & 2.0181899 & 2.0014218 & 2.0000719 & 2.0000129 & 2.0000014 & 2.0000000 \\
12 & 2.0113687 & 2.0006887 & 2.0000258 & 2.0000039 & 2.0000003 & 2.0000000 \\
13 & 2.0071054 & 2.0003336 & 2.0000093 & 2.0000012 & 2.0000001 & 2.0000000 \\
14 & 2.0044409 & 2.0001616 & 2.0000033 & 2.0000004 & 2.0000000 & 2.0000000 \\
15 & 2.0027756 & 2.0000783 & 2.0000012 & 2.0000001 & 2.0000000 & 2.0000000 \\
16 & 2.0017347 & 2.0000379 & 2.0000004 & 2.0000000 & 2.0000000 & 2.0000000 \\
17 & 2.0010842 & 2.0000184 & 2.0000002 & 2.0000000 & 2.0000000 & 2.0000000 \\
18 & 2.0006776 & 2.0000089 & 2.0000001 & 2.0000000 & 2.0000000 & 2.0000000 \\
19 & 2.0004235 & 2.0000043 & 2.0000000 & 2.0000000 & 2.0000000 & 2.0000000 \\
\hline
\end{tabular}

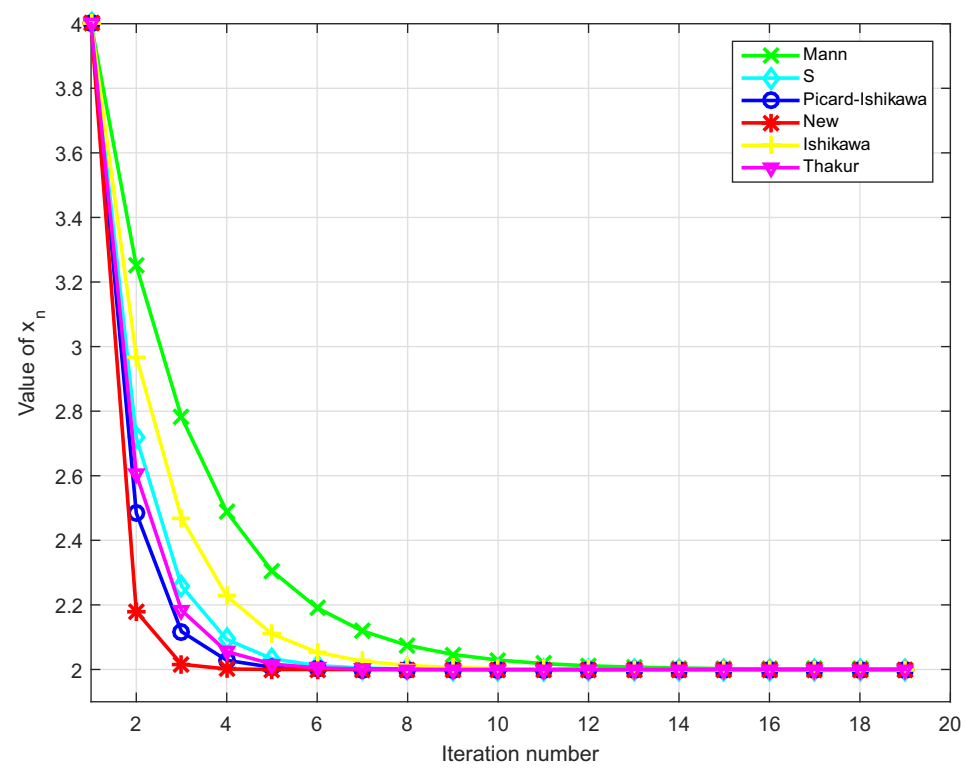

Figure 2. Graph corresponding to Table 2. 


\section{Application}

Existence theorem for fixed points of an operator is concerned with establishing sufficient conditions in which the operator will have solution, but does not necessarily show how to find it. On the other hand, the iteration method of fixed points is concerned with approximation or computation of sequences which converge to the solution of such operator.

In this section, we will approximate the solution of nonlinear Volterra delay integro-differential equations by utilizing the following iterative algorithm recently introduced by Ofem et al. [24]:

$$
\left\{\begin{array}{l}
x_{0} \in \mathcal{K}, \\
s_{n}=\left(1-v_{n}\right) x_{n}+v_{n} T x_{n}, \\
z_{n}=\left(1-u_{n}\right) T x_{n}+u_{n} T s_{n}, \quad \forall n \geq 1, \\
y_{n}=T z_{n}, \\
x_{n+1}=T y_{n},
\end{array}\right.
$$

where $\left\{u_{n}\right\}$ and $\left\{v_{n}\right\}$ are sequences in $(0,1)$.

Remark 2. We remark that the iterative algorithm (1.9) is the multivalued conversion of the iterative algorithm (6.1). It is shown in [24] that the iterative algorithm (6.1) has a better speed of convergence than S [3], Picard-S [12], Thakur [34] and M [36] iteration processes for single-valued generalized $\alpha$-nonexpansive mappings.

In particular, we will be interested in the following nonlinear Volterra delay integro-differential equation (VDIE):

$$
\begin{gathered}
x^{\prime}(t)=f\left(t, x(t), x(\hbar(t)), \int_{0}^{t} \wp(t, s, x(s), x(\hbar(s))) d s\right), \quad t \in I, \\
x(t)=\psi(t), \quad t \in[-r, 0]
\end{gathered}
$$

where $I=[0, k], k>0$ and $\psi \in C([-r, 0], \Re)$.

A function $x \in C([r, k], \Re) \cap C^{\prime}([0, k], \Re)$ that satisfies the equations (6.2)-(6.3) is called a solution of the initial value problem (6.2)-(6.3).

Suppose that the following conditions are performed:

$\left(M_{1}\right)$ Let $f \in C\left([0, k] \times \Re^{3}, \Re\right), \wp \in C\left([0, k] \times[0, k] \times \Re^{2}, \Re\right)$ and $\hbar \in C([0, k],[-r, k])$ be such that $\hbar(t) \leq t$

$\left(M_{2}\right)$ There exists constants $L_{f}, L_{\wp}>0$ such that

$$
\begin{gathered}
\left|f\left(t, \sharp_{1}, \sharp_{2}, \sharp_{3}\right)-f\left(t, b_{1}, b_{2}, b_{3}\right)\right| \leq L_{f}\left(\left|\sharp_{1}-b_{1}\right|+\left|\sharp_{2}-b_{2}\right|+\left|\sharp_{3}-b_{3}\right|\right) ; \\
\left|\wp\left(t, s, \sharp_{1}, \sharp_{2}\right)-\wp\left(t, s, b_{1}, b_{2}\right)\right| \leq L_{f}\left(\left|\sharp_{1}-b_{1}\right|+\left|\sharp_{2}-b_{2}\right|\right)
\end{gathered}
$$

for all $t, s \in I, \sharp_{i}, b_{i} \in \Re(i=1,2,3)$.

$\left(M_{3}\right) k L_{f}\left[2+L_{f} k\right]<1$.

$\left(M_{4}\right)$ The function $\phi:[-r, k] \rightarrow \Re_{+}$is positive, nondecreasing and continuous and there exists $\exists>0$ such that

$$
\int_{0}^{t} \phi(s) d s \leq \exists \phi(t), \quad t \in[0, k]
$$


Clearly, from assumption $\left(M_{1}\right)$, the initial value problem (6.2)-(6.3) is equivalent to the following integral equations:

$$
\begin{gathered}
x(t)=\psi(0)+\int_{0}^{t} f\left(s, x(s), x(\hbar(s)), \int_{0}^{s} \wp(s, \tau, x(\tau), x(\hbar(\tau))) d \tau\right) d s, \quad t \in I, \\
x(t)=\psi(t), \quad t \in[-r, 0] .
\end{gathered}
$$

The following existence result for initial value problem is due to Kucche and Shikhare [18].

Theorem 7. If the assumptions $\left(M_{1}\right)-\left(M_{4}\right)$ hold, then the problem (6.2)-(6.3) has a unique solution and the equation (6.2) is generalized Ulam-Hyers-Rassias stable with respect to the function $\phi$.

We now present our main result in section.

Theorem 8. Let $\left\{x_{n}\right\}$ be the iterative procedure (6.1) with $u_{n}, v_{n} \in(0,1)$ such that $\sum_{n=0}^{\infty} u_{n} v_{n}=\infty$. Suppose that the conditions $\left(M_{1}\right)-\left(M_{3}\right)$ are fulfilled. Then the initial value problem (6.2)-(6.3) has a unique solution, say, q in $C([r, k], \Re) \cap C^{\prime}([0, k], \Re)$ and $\left\{x_{n}\right\}$ converges to $q$.

P r o o f. Consider the Banach space $\mathscr{G}=C([-r, k], \Re)$ with Chebyshev norm $\|\cdot\|_{C}$. Let $\left\{x_{n}\right\}$ be an iterative sequence generated by the iteration process (6.1) for the operator $T: \mathscr{G} \rightarrow \mathscr{G}$ define by

$$
\begin{gathered}
T x(t)=\psi(0)+\int_{0}^{t} f\left(s, x(s), x(\hbar(s)), \int_{0}^{s} \wp(s, \tau, x(\tau), x(\hbar(\tau))) d \tau\right) d s, \quad t \in I, \\
T x(t)=\psi(t), \quad t \in[-r, 0] .
\end{gathered}
$$

Let $q$ stand for the fixed point of $T$. We now prove that $x_{n} \rightarrow q$ as $n \rightarrow \infty$. It obvious that for $t \in[-r, 0], x_{n} \rightarrow q$ as $n \rightarrow \infty$. Next for $t \in I$, we get

$$
\begin{aligned}
\left\|s_{n}-q\right\| & =\left\|\left(1-v_{n}\right) x_{n}+v_{n} T x_{n}-T q\right\| \\
& \leq\left(1-v_{n}\right)\left\|x_{n}-q\right\|+v_{n}\left\|T x_{n}-T q\right\| \\
& =\left(1-v_{n}\right)\left|x_{n}(t)-q(t)\right|+v_{n}\left|T\left(x_{n}\right)(t)-T(q)(t)\right| \\
& =\left(1-v_{n}\right)\left|x_{n}(t)-q(t)\right| \\
& +v_{n} \mid \psi(0)+\int_{0}^{t} f\left(s, x_{n}(s), x_{n}(\hbar(s)), \int_{0}^{s} \wp\left(s, \tau, x_{n}(\tau), x_{n}(\hbar(\tau))\right) d \tau\right) \\
& -\psi(0)-\int_{0}^{t} f\left(s, q(s), q(\hbar(s)), \int_{0}^{s} \wp(s, \tau, q(\tau), q(\hbar(\tau))) d \tau\right) \mid \\
& \leq\left(1-v_{n}\right)\left|x_{n}(t)-q(t)\right| \\
& +v_{n} \int_{0}^{t} L_{f}\left\{\max _{0 \leq d_{1} \leq s}\left|x_{n}\left(d_{1}\right)-q\left(d_{1}\right)\right|+\max _{0 \leq d_{1} \leq s}\left|x_{n}\left(\hbar\left(d_{1}\right)\right)-q\left(\hbar\left(d_{1}\right)\right)\right|\right. \\
& \left.+\int_{0}^{s} L_{\wp}\left[\max _{0 \leq d_{2} \leq \tau}\left|x_{n}\left(d_{2}\right)-q\left(d_{2}\right)\right|+\max _{0 \leq d_{1} \leq \tau}\left|x_{n}\left(\hbar\left(d_{2}\right)\right)-q\left(\hbar\left(d_{2}\right)\right)\right|\right] d \tau\right\} d s \\
& \leq\left(1-v_{n}\right)\left|x_{n}(t)-q(t)\right| \\
& +v_{n}^{t} \int_{0}^{t} L_{f}\left\{\max _{-r \leq d_{1} \leq k}\left|x_{n}\left(d_{1}\right)-q\left(d_{1}\right)\right|+\max _{-r \leq \tau_{1} \leq k}\left|x_{n}\left(\tau_{1}\right)-q\left(\tau_{1}\right)\right|\right. \\
& \left.+\int_{0}^{s} L_{\wp}\left[\max _{-r \leq d_{2} \leq k}\left|x_{n}\left(d_{2}\right)-q\left(d_{2}\right)\right|+\max _{-r \leq \tau_{2} \leq k}\left|x_{n}\left(\tau_{2}\right)-q\left(\tau_{2}\right)\right|\right] d \tau\right\} d s
\end{aligned}
$$


$\leq\left(1-v_{n}\right)\left\|x_{n}-q\right\|_{C}+v_{n} \int_{0}^{t} L_{f}\left\{2\left\|x_{n}-q\right\|_{C}+2 \int_{0}^{s} L_{\wp}\left\|x_{n}-q\right\|_{C} d \tau\right\} d s$

$\leq\left(1-v_{n}\right)\left\|x_{n}-q\right\|_{C}+v_{n} k L_{f}\left(2+L_{\wp} k\right)\left\|x_{n}-q\right\|_{C}$

$=\left[1-v_{n}\left(1-k L_{f}\left(2+L_{\wp} k\right)\right)\right]\left\|x_{n}-q\right\|_{C}$;

$\left\|z_{n}-q\right\| \leq\left(1-u_{n}\right)\left\|T x_{n}-T q\right\|+u_{n}\left\|T s_{n}-T q\right\|$

$=\left(1-u_{n}\right) \mid \int_{0}^{t} f\left(s, x_{n}(s), x_{n}(\hbar(s)), \int_{0}^{s} \wp\left(s, \tau, x_{n}(\tau), x_{n}(\hbar(\tau))\right) d \tau\right)$

$-\int_{0}^{t} f\left(s, q(s), q(\hbar(s)), \int_{0}^{s} \wp(s, \tau, q(\tau), q(\hbar(\tau))) d \tau\right)$

$+u_{n} \mid \int_{0}^{t} f\left(s, s_{n}(s), s_{n}(\hbar(s)), \int_{0}^{s} \wp\left(s, \tau, s_{n}(\tau), s_{n}(\hbar(\tau))\right) d \tau\right)$

$-\int_{0}^{t} f\left(s, q(s), q(\hbar(s)), \int_{0}^{s} \wp(s, \tau, q(\tau), q(\hbar(\tau))) d \tau\right) \mid$

$\leq\left(1-u_{n}\right) \int_{0}^{t} L_{f}\left\{\max _{0 \leq d_{1} \leq s}\left|x_{n}\left(d_{1}\right)-q\left(d_{1}\right)\right|+\max _{0 \leq d_{1} \leq s}\left|x_{n}\left(\hbar\left(d_{1}\right)\right)-q\left(\hbar\left(d_{1}\right)\right)\right|\right.$

$\left.+\int_{0}^{s} L_{\wp}\left[\max _{0 \leq d_{2} \leq \tau}\left|x_{n}\left(d_{2}\right)-q\left(d_{2}\right)\right|+\max _{0 \leq d_{1} \leq \tau}\left|x_{n}\left(\hbar\left(d_{2}\right)\right)-q\left(\hbar\left(d_{2}\right)\right)\right|\right] d \tau\right\} d s$

$+u_{n} \int_{0}^{t} L_{f}\left\{\max _{0 \leq d_{1} \leq s}\left|s_{n}\left(d_{1}\right)-q\left(d_{1}\right)\right|+\max _{0 \leq d_{1} \leq s}\left|s_{n}\left(\hbar\left(d_{1}\right)\right)-q\left(\hbar\left(d_{1}\right)\right)\right|\right.$

$\left.+\int_{0}^{s} L_{\wp}\left[\max _{0 \leq d_{2} \leq \tau}\left|s_{n}\left(d_{2}\right)-q\left(d_{2}\right)\right|+\max _{0 \leq d_{1} \leq \tau}\left|s_{n}\left(\hbar\left(d_{2}\right)\right)-q\left(\hbar\left(d_{2}\right)\right)\right|\right] d \tau\right\} d s$

$\leq\left(1-u_{n}\right) \int_{0}^{t} L_{f}\left\{\max _{-r \leq d_{1} \leq k}\left|x_{n}\left(d_{1}\right)-q\left(d_{1}\right)\right|+\max _{-r \leq \tau_{1} \leq k}\left|x_{n}\left(\tau_{1}\right)-q\left(\tau_{1}\right)\right|\right.$

$\left.+\int_{0}^{s} L_{\wp}\left[\max _{-r \leq d_{2} \leq k}\left|x_{n}\left(d_{2}\right)-q\left(d_{2}\right)\right|+\max _{-r \leq \tau_{2} \leq k}\left|x_{n}\left(\tau_{2}\right)-q\left(\tau_{2}\right)\right|\right] d \tau\right\} d s$

$+u_{n} \int_{0}^{t} L_{f}\left\{\max _{-r \leq d_{1} \leq k}\left|s_{n}\left(d_{1}\right)-q\left(d_{1}\right)\right|+\max _{-r \leq \tau_{1} \leq k}\left|s_{n}\left(\tau_{1}\right)-q\left(\tau_{1}\right)\right|\right.$

$\left.+\int_{0}^{s} L_{\wp}\left[\max _{-r \leq d_{2} \leq k}\left|s_{n}\left(d_{2}\right)-q\left(d_{2}\right)\right|+\max _{-r \leq \tau_{2} \leq k}\left|s_{n}\left(\tau_{2}\right)-q\left(\tau_{2}\right)\right|\right] d \tau\right\} d s$

$\leq\left(1-u_{n}\right) \int_{0}^{t} L_{f}\left\{2\left\|x_{n}-q\right\|_{C}+2 \int_{0}^{s} L_{\wp}\left\|x_{n}-q\right\|_{C} d \tau\right\} d s$

$+u_{n} \int_{0}^{t} L_{f}\left\{2\left\|s_{n}-q\right\|_{C}+2 \int_{0}^{s} L_{\wp}\left\|s_{n}-q\right\|_{C} d \tau\right\} d s$

$\leq\left(1-u_{n}\right) k L_{f}\left(2+L_{\wp} k\right)\left\|x_{n}-q\right\|_{C}+u_{n} k L_{f}\left(2+L_{\wp} k\right)\left\|s_{n}-q\right\|_{C}$

$=k L_{f}\left(2+L_{\wp} k\right)\left[\left(1-u_{n}\right)\left\|x_{n}-q\right\|_{C}+u_{n}\left\|s_{n}-q\right\|_{C}\right]$;

$$
\begin{aligned}
\left\|z_{n}-q\right\| & =\left\|T z_{n}-T q\right\| \\
& =\mid \int_{0}^{t} f\left(s, z_{n}(s), z_{n}(\hbar(s)), \int_{0}^{s} \wp\left(s, \tau, z_{n}(\tau), z_{n}(\hbar(\tau))\right) d \tau\right) \\
& -\int_{0}^{t} f\left(s, q(s), q(\hbar(s)), \int_{0}^{s} \wp(s, \tau, q(\tau), q(\hbar(\tau))) d \tau\right) \mid \\
& \leq \int_{0}^{t} L_{f}\left\{\max _{0 \leq d_{1} \leq s}\left|z_{n}\left(d_{1}\right)-q\left(d_{1}\right)\right|+\max _{0 \leq d_{1} \leq s}\left|z_{n}\left(\hbar\left(d_{1}\right)\right)-q\left(\hbar\left(d_{1}\right)\right)\right|\right.
\end{aligned}
$$




$$
\begin{aligned}
& \left.+\int_{0}^{s} L_{\wp}\left[\max _{0 \leq d_{2} \leq \tau}\left|z_{n}\left(d_{2}\right)-q\left(d_{2}\right)\right|+\max _{0 \leq d_{1} \leq \tau}\left|z_{n}\left(\hbar\left(d_{2}\right)\right)-q\left(\hbar\left(d_{2}\right)\right)\right|\right] d \tau\right\} d s \\
& \leq \int_{0}^{t} L_{f}\left\{\max _{-r \leq d_{1} \leq k}\left|z_{n}\left(d_{1}\right)-q\left(d_{1}\right)\right|+\max _{-r \leq \tau_{1} \leq k}\left|z_{n}\left(\tau_{1}\right)-q\left(\tau_{1}\right)\right|\right. \\
& \left.+\int_{0}^{s} L_{\wp}\left[\max _{-r \leq d_{2} \leq k}\left|z_{n}\left(d_{2}\right)-q\left(d_{2}\right)\right|+\max _{-r \leq \tau_{2} \leq k}\left|z_{n}\left(\tau_{2}\right)-q\left(\tau_{2}\right)\right|\right] d \tau\right\} d s \\
& \leq \int_{0}^{t} L_{f}\left\{2\left\|z_{n}-q\right\|_{C}+2 \int_{0}^{s} L_{\wp}\left\|z_{n}-q\right\|_{C} d \tau\right\} d s \\
& \leq k L_{f}\left(2+L_{\wp} k\right)\left\|z_{n}-q\right\|_{C} .
\end{aligned}
$$

$$
\begin{aligned}
\left\|x_{n+1}-q\right\| & =\left\|T y_{n}-T q\right\| \\
& =\mid \int_{0}^{t} f\left(s, y_{n}(s), y_{n}(\hbar(s)), \int_{0}^{s} \wp\left(s, \tau, y_{n}(\tau), y_{n}(\hbar(\tau))\right) d \tau\right) \\
& -\int_{0}^{t} f\left(s, q(s), q(\hbar(s)), \int_{0}^{s} \wp(s, \tau, q(\tau), q(\hbar(\tau))) d \tau\right) \mid \\
& \leq \int_{0}^{t} L_{f}\left\{\max _{0 \leq d_{1} \leq s}\left|y_{n}\left(d_{1}\right)-q\left(d_{1}\right)\right|+\max _{0 \leq d_{1} \leq s}\left|y_{n}\left(\hbar\left(d_{1}\right)\right)-q\left(\hbar\left(d_{1}\right)\right)\right|\right. \\
& \left.+\int_{0}^{s} L_{\wp}\left[\max _{0 \leq d_{2} \leq \tau}\left|y_{n}\left(d_{2}\right)-q\left(d_{2}\right)\right|+\max _{0 \leq d_{1} \leq \tau}\left|y_{n}\left(\hbar\left(d_{2}\right)\right)-q\left(\hbar\left(d_{2}\right)\right)\right|\right] d \tau\right\} d s \\
& \leq \int_{0}^{t} L_{f}\left\{\max _{-r \leq d_{1} \leq k}\left|y_{n}\left(d_{1}\right)-q\left(d_{1}\right)\right|+\max _{-r \leq \tau_{1} \leq k}\left|y_{n}\left(\tau_{1}\right)-q\left(\tau_{1}\right)\right|\right. \\
& \left.+\int_{0}^{s} L_{\wp}\left[\max _{-r \leq d_{2} \leq k}\left|y_{n}\left(d_{2}\right)-q\left(d_{2}\right)\right|+\max _{-r \leq \tau_{2} \leq k}\left|y_{n}\left(\tau_{2}\right)-q\left(\tau_{2}\right)\right|\right] d \tau\right\} d s \\
& \leq \int_{0}^{t} L_{f}\left\{2\left\|y_{n}-q\right\|_{C}+2 \int_{0}^{s} L_{\wp}\left\|y_{n}-q\right\|_{C} d \tau\right\} d s \\
& \leq k L_{f}\left(2+L_{\wp} k\right)\left\|y_{n}-q\right\|_{C} .
\end{aligned}
$$

Using (6.4), (6.5), (6.6) and (6.7), we get

$$
\left\|x_{n+1}-q\right\| \leq\left[k L_{f}\left(2+L_{\wp} k\right)\right]^{3}\left[1-u_{n} v_{n}\left(1-k L_{f}\left(2+L_{\wp} k\right)\right)\right]\left\|x_{n}-q\right\|_{C} .
$$

Using assumption $\left(M_{3}\right)$, we obtain

$$
\left\|x_{n+1}-q\right\| \leq\left[1-u_{n} v_{n}\left(1-k L_{f}\left(2+L_{\wp} k\right)\right)\right]\left\|x_{n}-q\right\|_{C} .
$$

Now define

$$
\sigma_{n}=u_{n} v_{n}\left(1-k L_{f}\left(2+L_{\wp} k\right)\right)<1,
$$

then $\sigma_{n} \in(0,1)$ such that $\sum_{0}^{\infty} \sigma_{n}=\infty$ and set $\theta_{n}=\left\|x_{n}-q\right\|_{C}$. Then (6.8) can be rewritten as

$$
\theta_{n+1}=\left(1-\sigma_{n}\right) \theta \text {. }
$$

Therefore, all the conditions of Lemma 2 are satisfied. Hence, $\lim _{n \rightarrow \infty}\left\|x_{n}-q\right\|=0$.

Now, we furnish the following example in support of the above claims in Theorem 8 .

Example 4. Consider the following nonlinear delay Volterra integro-differential equations:

$$
\begin{gathered}
x^{\prime}(t)=1+\frac{t x(t)}{25}-\frac{7 t x(\hbar(t))}{50}+\frac{1}{5} \int_{0}^{t} \frac{1}{10}[x(s)-x(\hbar(s))] d s, \quad t \in[0,3], \\
x(t)=0, \quad t \in[-1,0],
\end{gathered}
$$

where $\hbar(t)=t / 3, t \in[0,3]$. Obviously, we have that $\hbar(t)=t / 3 \leq t, t \in[0,3]$. 
(i) Define $\wp:[0,3] \times[0,3] \times \Re \times \Re \rightarrow \Re$ by

$$
\wp(t, s, x(s), x(\hbar(s)))=\frac{1}{5}[x(s)-x(\hbar(s))], \quad t, s \in[0,3] .
$$

Then for $t, s \in[0,3]$ and $\sharp_{i}, b_{i} \in \Re(i=1,2)$, we have

$$
\left|\wp\left(t, s, \sharp_{1}, \sharp_{2}\right)-\wp\left(t, s, b_{1}, b_{2}\right)\right| \leq \frac{1}{5}\left(\left|\sharp_{1}-b_{1}\right|+\left|\sharp_{2}-b_{2}\right|\right) .
$$

(ii) Define $f:[0,3] \times \Re \times \Re \times \Re \rightarrow \Re$ by

$$
\begin{gathered}
f\left(t, x(t), x(\hbar(t)), \int_{0}^{s} \wp(t, s, x(s), x(\hbar(s))) d s\right) \\
=1+\frac{t x(t)}{25}-\frac{7 t x(\hbar(t))}{50}+\frac{1}{10} \int_{0}^{t} \frac{1}{5}[x(s)-x(\hbar(s))] d s \\
=1+\frac{t x(t)}{25}-\frac{7 t x(\hbar(t))}{50}+\frac{1}{10} \int_{0}^{t} \wp(t, s, x(s), x(\hbar(s))) d s, \quad t \in[0,3] .
\end{gathered}
$$

Then, for any $t, s \in[0,3]$ and $\sharp_{i}, b_{i} \in \Re(i=1,2,3)$, we have

$$
\begin{aligned}
\left|f\left(t, \sharp_{1}, \sharp_{2}, \sharp_{3}\right)-f\left(t, b_{1}, b_{2}, b_{3}\right)\right| & \leq \frac{1}{25}\left|\sharp_{1}-b_{2}\right|+\frac{7}{50}\left|\sharp_{2}-b_{2}\right|+\frac{1}{10}\left|\sharp_{3}-b_{3}\right| \\
& \leq \frac{7}{50}\left(\left|\sharp_{1}-b_{2}\right|+\left|\sharp_{2}-b_{2}\right|+\left|\sharp_{3}-b_{3}\right|\right) .
\end{aligned}
$$

Thus the above defined functions $f$ and $\wp$ satisfy the assumptions $\left(M_{1}\right)$ and $\left(M_{2}\right)$ with $L_{f}=7 / 50$, $L_{\wp}=1 / 10$. Further, we see that

$$
k L_{f}\left(2+k L_{h}\right)=3 \cdot \frac{7}{50}\left(2+3 \cdot \frac{1}{10}\right)=\frac{483}{500}<1 .
$$

Thus condition $\left(M_{3}\right)$ holds. Now, if we take $u_{n}=n /(n+1)$ and $v_{n}=1 / n$, it follows that

$$
\sum_{n=0}^{\infty} u_{n} v_{n}=\infty \text {. }
$$

In addition, we notice that the exact solution of the problem (6.2)-(6.3) is the function

$$
x(t)= \begin{cases}t, & \text { if } t \in[0,3], \\ 0, & \text { if } t \in[-1,0] .\end{cases}
$$

Indeed, for $x(t)=t, t \in[0,3]$ and $\hbar(t)=t / 3, t \in[0,3]$, we get

$$
1+\frac{t x(t)}{25}-\frac{7 t x(\hbar(t))}{50}+\frac{1}{5} \int_{0}^{t} \frac{1}{10}[x(s)-x(\hbar(s))] d s=1+\frac{t^{2}}{25}-\frac{7 t^{2}}{150}+\frac{1}{5} \int_{0}^{t} \frac{1}{10}\left[s-\frac{s}{3}\right] d s=1=x^{\prime}(t) .
$$

Thus, all the conditions of Theorem 8 are fulfilled. Hence, Theorem 8 is applicable.

Remark 3. For any fixed $r>0$, define

$$
\hbar_{1}(t)=t-r, \quad t \in[0, k]
$$


Then we get the following special case of the Problem (6.2)-(6.3) as follows:

$$
\begin{gathered}
x^{\prime}(t)=f_{1}\left(t, x(t), x(t-r), \int_{0}^{t} \wp_{1}(t, s, x(s), x(t-r)) d s\right), \quad t \in[0, k], \\
x(t)=\psi(t), \quad t \in[-r, 0],
\end{gathered}
$$

which is the initial value problem for a nonlinear Volterra integro-differential equation.

The approximation of solution the problem (6.9)-(6.10) has been studied by several authors for $\wp_{1}(t, s, x(s), x(t-r))=0$ (see for example [4, 9, 12, 23, 25-27] and the references there in). Hence, our result in Theorem 8 generalizes the corresponding results in $[4,9,12,23,25-27]$ and several others.

\section{Conclusion}

In this paper, we have studied the stability result of our newly introduced iterative algorithm (1.9) for a wider concept of stability known as $w^{2}$-stability instead of the simple notion of stability considered in $[15,24,28]$. A numerical example is also used to support the analytical proof of our stability theorem. We have also proved the weak and strong convergence theorems of our new iterative algorithm (1.9) for fixed points of multivalued generalized $\alpha$-nonexpansive mappings. In addition, a numerical experiment was also carried out to illustrate the advantage of our iterative method over some well known iterative methods in the literature. Further, as application of our new iterative algorithm (1.9), we approximated the solution of nonlinear Volterra delay integro-differential equations (6.2)-(6.3). A nontrivial example of a nonlinear Volterra delay integro-differential equation which satisfies all the mild conditions used in obtaining our result has been provided. We have also seen that the class of delay differential equation studied in $[4,9,12,23,25-27]$ is a special case of the class nonlinear Volterra delay integro-differential equation considered in this article. Hence, our results generalize, improve and unify the corresponding results in $[4,9,12,15,23-28]$ and several others in the existing literature.

\section{Acknowledgements}

The authors are grateful to the reviewers and editors for their useful comments which helped to improve this work.

\section{REFERENCES}

1. Abbas M., Nazir T. A new faster iteration process applied to constrained minimization and feasibility problems. Mat. Vesnik, 2014. Vol. 66, No. 2. P. 223-234. URL: http://hdl.handle.net/2263/43663

2. Abkar A., Eslamian M. A fixed point theorem for generalized nonexpansive multivalued mappings. Fixed Point Theory, 2011. Vol. 12, No. 2. P. 241-246.

3. Agarwal R.P., O'Regan D., Sahu D. R. Iterative construction of fixed points of nearly asymptotically nonexpansive mappings. J. Nonlinear Convex Anal., 2007. Vol. 8, No. 1. P. 61-79.

4. Ali F., Ali J, Nieto J. J. Some observations on generalized non-expansive mappings with an application. Comp. Appl. Math., 2020. Vol. 39. Art. no. 74. P. 1-20. DOI: 10.1007/s40314-020-1101-4

5. Aoyama K., Kohsaka F. Fixed point theorem for $\alpha$-nonexpansive mappings in Banach spaces. Nonlinear Anal., 2011. Vol. 74, No. 13. P. 4387-4391. DOI: 10.1016/j.na.2011.03.057

6. Berinde V. Iterative Approximation of Fixed Points. Berlin, Heidelberg: Springer, 2007. 326 p. DOI: $10.1007 / 978-3-540-72234-2$

7. Browder F. E. Nonexpansive nonlinear operators in a Banach space. Proc. Nat. Acad. Sci. USA., 1965. Vol. 54, No. 4. P. 1041-1044. DOI: 10.1073/pnas.54.4.1041 
8. Cardinali T., Rubbioni P. A generalization of the Caristi fixed point theorem in metric spaces. Fixed Point Theory, 2010. Vol. 11, No. 1. P. 3-10.

9. Garodia C., Uddin I. A new fixed point algorithm for finding the solution of a delay differential equation. AIMS Mathematics, 2020. Vol. 5, No. 4. P. 3182-3200. DOI: 10.3934/math.2020205

10. Göhde D. Zum Prinzip der kontraktiven Abbildung. Math. Nachr., 1965. Vol. 30, No. 3-4. P. $251-258$. (in German) DOI: 10.1002/mana.19650300312

11. Gunduz B., Alagoz O., Akbulut S. Convergence theorems of a faster iteration process including multivalued mappings with analytical and numerical examples. Filomat, 2018. Vol. 32, No. 16. P. 5665-5677. DOI: $10.2298 /$ FIL1816665G

12. Gürsoy F., Karakaya V. A Picard-S Hybrid Type Iteration Method for Solving a Differential Equation with Retarded Argument. 2014. 16 p. arXiv:1403.2546v2 [math.FA]

13. Harder A.M. Fixed Point Theory and Stability Results for Fixed Point Iteration Procedures. Ph.D. thesis. Missouri: University of Missouri-Rolla, 1987. 70 p.

14. Harder A. M., Hicks T.L. A stable iteration procedure for nonexpansive mappings. Math. Japonica, 1988. Vol. 33, No. 5. P. 687-692.

15. Iqbal H., Abbas M., Husnine S. M. Existence and approximation of fixed points of multivalued generalized $\alpha$-nonexpansive mappings in Banach spaces. Numer. Algor., 2020. Vol. 85. P. 1029-1049. DOI: $10.1007 / \mathrm{s} 11075-019-00854-\mathrm{z}$

16. Ishikawa S. Fixed points by a new iteration method. Proc. Amer. Math. Soc., 1994. Vol. 44. P. $147-150$. DOI: $10.2307 / 2039245$

17. Kirk W. A. A fixed point theorem for mappings which do not increase distance. Amer. Math. Monthly, 1965. Vol. 72, No. 9. P. 1004-1006. DOI: 10.2307/2313345

18. Kucche K. D., Shikhare P. U. Ulam Stabilities for nonlinear Volterra delay integro-differential equations. J. Contemp. Math. Anal., 2019. Vol. 54, No. 5. P. 276-287. DOI: $10.3103 /$ S1068362319050042

19. Mann W. R. Mean value methods in iteration. Proc. Amer. Math. Soc., 1953. Vol. 4. P. 506-510. DOI: 10.1090/S0002-9939-1953-0054846-3

20. Markin J. A fixed point theorem for set valued mappings. Bull. Amer. Math. Soc., 1968. Vol. 74, No. 1. P. 639-640.

21. Nadler S. B. Multi-valued contraction mappings. Pacific J. Math., 1969. Vol. 30, No. 2. P. $475-488$. DOI: $10.2140 /$ pjm.1969.30.475

22. Noor M. A. New approximation schemes for general variational inequalities. J. Math. Anal. Appl., 2000. Vol. 251, No. 1. P. 217-229. DOI: 10.1006/jmaa.2000.7042

23. Ofem A.E., Igbokwe D. I. An efficient iterative method and its applications to a nonlinear integral equation and a delay differential equation in Banach spaces. Turkish J. Ineq., 2020. Vol. 4, No. 2. P. 79-107.

24. Ofem A.E., Udofia U.E., Igbokwe D. I. New iterative algorithm for solving constrained convex minimization problem and split feasibility problem. Eur. J. Math. Anal., 2021. Vol. 1, No. 2. P. 106-132. DOI: $10.28924 / \mathrm{ada} / \mathrm{ma} .1 .106$

25. Ofem A.E., Udofia U.E. Iterative solutions for common fixed points of nonexpansive mappings and strongly pseudocontractive mappings with applications. Canad. J. Appl. Math., 2021. Vol. 3, No. 1. P. $18-36$.

26. Okeke G. A. Convergence analysis of the Picard-Ishikawa hybrid iterative process with applications. Afr. Mat., 2019. Vol. 30, No. 5-6. P. 817-835. DOI: 10.1007/s13370-019-00686-z

27. Okeke G.A., Abbas M.A. A solution of delay differential equations via Picard-Krasnoselskii hybrid iterative process. Arab. J. Math., 2017. Vol. 6. P. 21-29. DOI: 10.1007/s40065-017-0162-8

28. Okeke G.A., Abbas M.A., de la Sen M. Approximation of the fixed point of multivalued quasinonexpansive mappings via a faster iterative. Process with applications. Discrete Dyn. Nat. Soc., 2020. Vol. 2020. Art. no. 8634050. P. 1-11. DOI: 10.1155/2020/8634050

29. Pant D., Shukla R. Approximating fixed points of generalized $\alpha$-nonexpansive mappings in Banach spaces. Numer. Funct. Anal. Optim., 2017. Vol. 38, No. 2. P. $248-266$. DOI: $10.1080 / 01630563.2016 .1276075$

30. Schu J. Weak and strong convergence to fixed points of asymptotically nonexpansive mappings. Bull. Aust. Math. Soc., 1991. Vol. 43, No. 1. P. 153-159. DOI: 10.1017/S0004972700028884 
31. Senter H. F., Dotson W. G. Approximating fixed points of nonexpansive mappings. Proc. Amer. Math. Soc., 1974. Vol. 44, No. 2. P. 375-380. DOI: 10.2307/2040440

32. Song Y., Cho Y. J. Some notes on Ishikawa iteration for multivalued mappings. Bull. Korean Math. Soc., 2011. Vol. 48, No. 3. P. 575-584. DOI: 10.4134/BKMS.2011.48.3.575

33. Suzuki T. Fixed point theorems and convergence theorems for some generalized nonexpansive mappings. J. Math. Anal. Appl., 2008. Vol. 340, No. 2. P. 1088-10995. DOI: 10.1016/j.jmaa.2007.09.023

34. Thakur B.S., Thakur D., Postolache M. A new iteration scheme for approximating fixed points of nonexpansive mappings. Filomat, 2016. Vol. 30, No. 10. P. 2711-2720. DOI: 10.2298/FIL1610711T

35. Timis I. On the weak stability of Picard iteration for some contractive type mappings and coincidence theorems. Int. J. Comput. Appl., 2012. Vol. 37, No. 4. P. 9-13.

36. Ullah K., Arshad M. Numerical reckoning fixed points for Suzuki's generalized nonexpansive mappings via new iteration process. Filomat, 2018. Vol. 32. P. 187-196. DOI: 10.2298/FIL1801187U

37. Weng X. Fixed point iteration for local strictly pseudo-contractive mapping. Proc. Amer. Math. Soc., 1991. Vol. 113. P. 727-731. DOI: 10.1090/S0002-9939-1991-1086345-8 